OUTP-95-385

ISAS/EP $/ 95 / 78$

\title{
The Spin-Spin Correlation Function in the Two-Dimensional Ising Model in a Magnetic Field at $T=T_{c}$
}

\author{
G. Delfino \\ Theoretical Physics, University of Oxford \\ 1 Keble Road, Oxford OX1 3NP, United Kingdom \\ and \\ G. Mussardo \\ International School for Advanced Studies, \\ and \\ Istituto Nazionale di Fisica Nucleare \\ 34014 Trieste, Italy
}

\begin{abstract}
The form factor bootstrap approach is used to compute the exact contributions in the large distance expansion of the correlation function $\langle\sigma(x) \sigma(0)>$ of the twodimensional Ising model in a magnetic field at $T=T_{c}$. The matrix elements of the magnetization operator $\sigma(x)$ present a rich analytic structure induced by the (multi) scattering processes of the eight massive particles of the model. The spectral representation series has a fast rate of convergence and perfectly agrees with the numerical determination of the correlation function.
\end{abstract}




\section{Introduction}

Over the past few years, considerable progress has been made in the use of conformal invariance methods and scattering theory for the understanding of the critical points and the nearby scaling region of two-dimensional statistical models (see, for instance [1, 2]). At the critical points, the correlation functions of the statistical models fall into a scaleinvariant regime and their computation may be achieved by solving the linear differential equations obtained by the representation theory of the infinite-dimensional conformal symmetry [3]. The situation is different away from criticality. The scaling region may be described in terms of the relevant deformations of the fixed point actions. These deformations destroy the long-range fluctuations of the critical point and the associated quantum field theories are usually massive. If an infinite number of conserved charges survive the deformation of the critical point action, the corresponding QFT can be efficiently characterized (on-shell) by the relativistic scattering processes of the massive excitations. In this case, the integrals of motion severely restrict the bound state structure of the theory and force the $S$-matrix to be elastic and factorizable [4, 5]. Once the exact $S$-matrix of a model is known, one can proceed further and investigate the off-shell behaviour of the theory by means of the Form Factor approach [6, 7]. This consists in computing matrix elements of the local fields on the set of asymptotic states and reconstructing their correlation functions in terms of the spectral density representation. As argued in [8], and also confirmed by the explicit solution of several two-dimensional massive QFT [9, 10, 11, 12, 13], a general property of the spectral series of massive integrable QFT is their very fast rate of convergence for all distance scales. This important quality of the spectral representation series may be regarded as the key point to the success of the Form Factor approach.

The aim of this paper is to apply the Form Factor approach and compute the spinspin correlation function $G(x)=<\sigma(x) \sigma(0)>$ of the Ising model in a magnetic field $h$ at $T=T_{c}$ (in the sequel, this model will be referred to as IMMF). For the other integrable deformation of the critical Ising model, i.e. thermal, the spin-spin correlation function has been exactly determined in [14, 15] and this result, together with its remarkable connection with the Painleve' functions [16, 17], may be regarded as one of the main accomplishments in statistical mechanics. On the other hand, very little is known about the spin-spin correlation function for $h \neq 0$ at $T=T_{c}$ whose determination has been a long-standing problem of statistical mechanics. As we will show in this paper, the computation of $G(x)$ can now be approached analytically, thanks to the scattering formulation of the model

proposed by Zamolodchikov [4]. Apart from the importance of computing $G(x)$ itself, there are other related theoretical issues which render this calculation instructive.

The first issue concerns the rich structure of bound states and higher-order poles of 
the $S$-matrix. Higher-order poles of the $S$-matrix of two-dimensional field theories can be naturally interpreted as singularities associated to multi-scattering processes [18, 19, 20]. On this basis, one would expect a similar hierarchy of higher-order poles in the form factors as well. There is, however, an important difference between the pole structures of the $S$-matrix and those of the form factors. In fact, the $S$-matrix contains simultaneously information about the $s$-channel as well as the $u$-channel. Correspondingly, the poles of the $S$-matrix are always arranged in pairs, with their positions located in crossing symmetrical points. This is in concordance with the two different ways of looking at a scattering process, i.e. in the direct or in the crossed channel. On the contrary, the $u$-variable plays no role in the calculation of the form factors as these only depend on the $s$-variables of all subchannels of the asymptotic states on which the matrix elements are computed. The absence of $u$-channel singularities in the form factors implies that their analytic structure may be different from that of the $S$-matrix and it is therefore an interesting problem to understand how the higher-order poles enter the form factor calculation.

The second theoretical issue that emerges from the computation of the spin-spin correlation function in the IMMF is a careful reconsideration of the so-called minimality prescription, which is usually invoked for computing the form factors. Briefly stated, this consists in assuming that the form factors have the minimal analytic structure, compatible with the nature of the operator and the bound state pattern of the scattering theory. So far, this prescription has been successfully applied to solve several two-dimensional QFT as, for instance, those of refs. 9, 10, 11, 12, 13, despite the fact that the theoretical reason of its validity was lacking. One of the basic motivations why minimality is often adopted is because frequently the asymptotic behaviour of the matrix elements is not easy to determine. In this paper, we will present a simple argument which will allow us to place a quite restrictive upper bound on the high energy limit of form factors. Using this criterion, one can see that the minimality prescription is violated in the IMMF and therefore extra polynomials in the variable $s$ have to be included in the matrix elements of the field $\sigma(x)$. These polynomials, nevertheless, can be uniquely determined since the scattering theory always provides a sufficient number of constraints. As a matter of fact, the equations which fix the extra polynomials are usually overdetermined and this gives rise to self-consistency conditions which are indeed fulfilled by the IMMF form factors.

The last point we would like to mention is the successful comparison of our theoretical determination of $\langle\sigma(x) \sigma(0)>$ with numerical simulations. These simulations have been carried out in the last few years by two different groups [22, 23, 24] and in particular, a collection of high-precision numerical estimates of the spin-spin correlation function of the IMMF, for different values of the magnetic field and different sizes of the lattice, can 
be found in [23]. Although there is no doubt about the actual existence of higher-mass particles, which can be easily extracted by transfer matrix diagonalization of the IMMF [25] or directly from the lattice [26], it was quite difficult to see the presence of these higher-mass states from the analysis of the numerical data of the spin-spin correlation function. Namely, a best fit of the two-point function seemed to be compatible with the only contribution of the fundamental particle [22], a result which appears intriguing. In fact, from a theoretical point of view there is no reason why decoupling of highermass particles should occur in the spin-spin correlator since the IMMF has apparently no selection rules related to any symmetry. Indeed, as we will see, the magnetization field $\sigma(x)$ couples to all particles of the theory and it is only the small values of the relative couplings which could be responsible for a possible misleading interpretation of the numerical results.

The paper is organized as follows. In Section 2, we briefly outline the main features of scattering theory of the IMMF, the exact mass spectrum of the model and some other quantities which will prove useful in the sequel of the paper. In Section 3, we analyse the Form Factor approach and the properties of the spectral representation series of the correlation functions. In Section 4, a general constraint on the asymptotic behaviour of the form factors is introduced and applied to the computation of the matrix elements of the magnetization operator $\sigma(x)$ of the IMMF. We also discuss the occurrence and the interpretation of higher order poles in the form factors. Comparison of our theoretical results versus numerical simulations, as well as the saturation of the sum-rules satisfied by the spin-spin correlation function, are presented in Section 5 . The paper also contains three appendices. In Appendix A we discuss the general bootstrap approach to the computation of the form factors of the model. Appendix B gathers useful mathematical formulas to deal with the monodromy properties of the matrix elements. Appendix $\mathrm{C}$ presents specific examples of matrix elements with singularities associated to higher order poles in the scattering amplitudes.

\section{$2 \quad$ Scattering theory}

In the continuum limit, the IMMF may be regarded as a perturbed CFT. At the critical point, ( $T=T_{c}$ and $\left.h=0\right)$, the Ising model is described by the conformal minimal model $\mathcal{M}_{3,4}$ with central charge $C=\frac{1}{2}$ [3]. There are three conformal families in the model, those of the identity, magnetization and energy operators, denoted respectively as $[I=(0,0)]$, $\left[\sigma=\left(\frac{1}{16}, \frac{1}{16}\right)\right]$ and $\left[\epsilon=\left(\frac{1}{2}, \frac{1}{2}\right)\right]$, where the numbers $\left(\Delta_{i}, \Delta_{i}\right)$ in the round brackets are their conformal weights. We can move the system away from criticality by modifying the 
action $\mathcal{A}_{0}$ of the critical point as

$$
\mathcal{A}=\mathcal{A}_{0}+h \int d^{2} x \sigma(x)
$$

For small values of the magnetic field, the system is still at $T=T_{c}$. However, the coupling to the magnetic field induces a mass scale $M(h)$ in the problem and destroys the long-range fluctuations of the critical point. Using the action (2.1), or the corresponding lattice Hamiltonian, one can in principle analyse the properties of the model in terms of a perturbative expansion in $h$ [27, 28]. There are, however, some important questions which cannot be easily addressed by using such perturbative formulation, as, for instance, the determination of the exact mass spectrum of the model or the computation of its correlation functions. In order to answer such questions, we have to rely on a nonperturbative approach that exploits the most important feature of the dynamics of the model, namely its integrability. The IMMF has, in fact, an infinite number of conserved charges of spin $s=1,7,11,13,17,19,23,29(\bmod 30)$. As shown in a remarkable paper by Zamolodchikov [4], the existence of these conserved charges allows one to define a self-consistent scattering theory which provides an alternative route to the perturbative formulation. Let us briefly outline the main results discussed in [4].

The scattering theory which describes the scaling limit of the IMMF contains eight different species of self-conjugated particles $A_{a}, a=1, \ldots, 8$ with masses

$$
\begin{aligned}
& m_{1}=M(h), \\
& m_{2}=2 m_{1} \cos \frac{\pi}{5}=(1.6180339887 . .) m_{1}, \\
& m_{3}=2 m_{1} \cos \frac{\pi}{30}=(1.9890437907 . .) m_{1}, \\
& m_{4}=2 m_{2} \cos \frac{7 \pi}{30}=(2.4048671724 . .) m_{1}, \\
& m_{5}=2 m_{2} \cos \frac{2 \pi}{15}=(2.9562952015 . .) m_{1}, \\
& m_{6}=2 m_{2} \cos \frac{\pi}{30}=(3.2183404585 . .) m_{1}, \\
& m_{7}=4 m_{2} \cos \frac{\pi}{5} \cos \frac{7 \pi}{30}=(3.8911568233 . .) m_{1}, \\
& m_{8}=4 m_{2} \cos \frac{\pi}{5} \cos \frac{2 \pi}{15}=(4.7833861168 . .) m_{1},
\end{aligned}
$$

Within the standard CFT normalization of the magnetization operator, fixed by the equation

$$
<\sigma(x) \sigma(0)>=\frac{1}{|x|^{\frac{1}{4}}}, \quad|x| \rightarrow 0
$$

the overall mass scale $M(h)$ has been exactly determined in 33,

$$
M(h)=\mathcal{C} h^{\frac{8}{15}}
$$


where

$$
\mathcal{C}=\frac{4 \sin \frac{\pi}{5} \Gamma\left(\frac{1}{5}\right)}{\Gamma\left(\frac{2}{3}\right) \Gamma\left(\frac{8}{15}\right)}\left(\frac{4 \pi^{2} \Gamma\left(\frac{3}{4}\right) \Gamma^{2}\left(\frac{13}{16}\right)}{\Gamma\left(\frac{1}{4}\right) \Gamma^{2}\left(\frac{3}{16}\right)}\right)^{\frac{4}{5}}=4.40490858 \ldots
$$

The scattering processes in which the eight particles are involved are completely elastic (the final state contains exactly the same particles as the initial one with unchanged momenta) and, due to the factorization of multiparticle scattering amplitudes induced by integrability, they are entirely characterised by the two-particle amplitudes $S_{a b}$ (Figure $1)$. These are functions of the relativistic invariant Mandelstam variable $s=\left(p_{a}+p_{b}\right)^{2}$ or, equivalently, of $u=\left(p_{a}-p_{b}\right)^{2}$. $S_{a b}$ has a branch root singularity in the variable $s$ at the threshold $s=\left(m_{a}+m_{b}\right)^{2}$. By crossing symmetry, an analogous branch point also appears at the threshold of the $u$-channel, namely at $s=\left(m_{a}-m_{b}\right)^{2}$ (Figure 2). Those are the only branch cuts of the $S$-matrix, due to the elastic nature of the scattering processes. The other possible singularities of the scattering amplitudes $S_{a b}$ are simple and higher-order poles in the interval $\left(m_{a}-m_{b}\right)^{2}<s<\left(m_{a}+m_{b}\right)^{2}$ which are related to the bound state structure.

An important simplification in the analysis of the analytic structure of the $S$-matrix comes from the parameterization of the external momenta in terms of the rapidity variable $\theta$, i.e. $p_{a}^{0}=m_{a} \cosh \theta_{a}, p_{a}^{1}=m_{a} \sinh \theta_{a}$. The mapping $s\left(\theta_{a b}\right)=m_{a}^{2}+m_{b}^{2}+2 m_{a} m_{b} \cosh \theta_{a b}$, where $\theta_{a b}=\theta_{a}-\theta_{b}$, or equivalently $u\left(\theta_{a b}\right)=s\left(i \pi-\theta_{a b}\right)=m_{a}^{2}+m_{b}^{2}-2 m_{a} m_{b} \cosh \theta_{a b}$, transforms the amplitudes $S_{a b}$ into meromorphic functions $S_{a b}\left(\theta_{a b}\right)$, which satisfy the equations

$$
\begin{gathered}
S_{a b}(\theta) S_{a b}(-\theta)=1 ; \\
S_{a b}(\theta)=S_{a b}(i \pi-\theta),
\end{gathered}
$$

expressing the unitarity and the crossing symmetry of the theory, respectively. The simple poles of $S_{a b}(\theta)$ with positive residues are related to bound state propagation in the $s$ channel, as shown in Figure 3, whereas those with negative residues are associated to bound states in the $u$-channel. Suppose that the particle $A_{c}$ with mass squared

$$
m_{c}^{2}=m_{a}^{2}+m_{b}^{2}+2 m_{a} m_{b} \cos u_{a b}^{c}, \quad u_{a b}^{c} \in(0, \pi)
$$

is a stable bound state in the $s$-channel of the particles $A_{a}$ and $A_{b}$. In the vicinity of the resonance angle $\theta=i u_{a b}^{c}$, the amplitude becomes

$$
S_{a b}\left(\theta \simeq i u_{a b}^{c}\right) \simeq \frac{i\left(\Gamma_{a b}^{c}\right)^{2}}{\theta-i u_{a b}^{c}},
$$

with $\Gamma_{a b}^{c}$ denoting the three-particle couplingt. Since the bootstrap principle gives the

\footnotetext{
${ }^{1}$ Note that the $S$-matrix cannot determine the three-particle couplings but only their square. This results in an ambiguity for the sign of all $\Gamma_{a b}^{c}$ which can be solved by defining a consistent set of form factors.
} 
possibility to consider the bound states on the same footing as the asymptotic states, the amplitudes $S_{a b}$ are related each other by the functional equations [4, 5]

$$
S_{i l}(\theta)=S_{i j}\left(\theta+i \bar{u}_{j l}^{k}\right) S_{i k}\left(\theta-i \bar{u}_{l k}^{j}\right)
$$

$\left(\bar{u}_{a b}^{c} \equiv \pi-u_{a b}^{c}\right)$. For the IMMF, the solution of eqs. (2.6), (2.7) and (2.10) are given by

$$
S_{a b}(\theta)=\prod_{\alpha \in \mathcal{A}_{a b}}\left(f_{\alpha}(\theta)\right)^{p_{\alpha}}
$$

where

$$
f_{\alpha}(\theta) \equiv \frac{\tanh \frac{1}{2}(\theta+i \pi \alpha)}{\tanh \frac{1}{2}(\theta-i \pi \alpha)} .
$$

The sets of numbers $\mathcal{A}_{a b}$ and their multiplicities $p_{\alpha}$, specifying the amplitudes (2.11), can be found in Table 1 1 . In order to correctly interpret this collection of data, notice that the functions $f_{\alpha}(\theta)$ satisfy the equation $f_{\alpha}(\theta)=f_{1-\alpha}(\theta)$ as well as $f_{\alpha}(\theta)=f_{\alpha}(i \pi-\theta)$. Hence, they have two poles located at the crossing symmetrical positions $\theta=i \pi \alpha$ and $\theta=i \pi(1-\alpha)$. Therefore, the poles of the $S$-matrix of the IMMF will always appear in pairs.

Applying eq. (2.9) to the simple poles with positive residues at $\theta=\frac{2 \pi i}{3}, \theta=\frac{2 \pi i}{5}$ and $\theta=\frac{i \pi}{15}$ in $S_{11}(\theta)$ (related to the bound states $A_{1}, A_{2}$ and $A_{3}$ respectively), we can extract

$$
\begin{aligned}
& \Gamma_{11}^{1}=\sqrt{\frac{2 \tan \frac{2 \pi}{3} \tan \frac{8 \pi}{15} \tan \frac{11 \pi}{30}}{\tan \frac{2 \pi}{15} \tan \frac{3 \pi}{10}}}=10.990883 . . \\
& \Gamma_{11}^{2}=\sqrt{\frac{2 \tan \frac{2 \pi}{5} \tan \frac{8 \pi}{15} \tan \frac{7 \pi}{30}}{\tan \frac{2 \pi}{15} \tan \frac{\pi}{6}}}=14.322681 . . \\
& \Gamma_{11}^{3}=\sqrt{\frac{2 \tan \frac{\pi}{15} \tan \frac{11 \pi}{30} \tan \frac{7 \pi}{30}}{\tan \frac{\pi}{6} \tan \frac{3 \pi}{10}}}=1.0401363 . .
\end{aligned}
$$

Other three-particle couplings can be obtained similarly. In addition to simple poles, the $S$-matrix of the IMMF presents higher-order poles due to multi-scattering processes 5 . The odd order poles correspond to bound state poles while those of even order do not. Their appearance is an unavoidable consequence of the iterative application of the bootstrap equations (2.10). In relation with the calculation of the Form Factors, some of these poles will be considered in Section 4 and in Appendix C.

\footnotetext{
${ }^{2}$ Note that the numbers $\mathcal{A}_{a b}$ of Table 1 should be read in units of $\frac{1}{30}$.

${ }^{3}$ A detailed analysis of the nature of higher-order poles in the $S$-matrix of the $(1+1)$ integrable theories can be found in [18, 19, 20].
} 


\section{Correlation Functions and Form Factors}

Once the spectrum and the $S$-matrix are known, we can investigate the off-shell behaviour of the theory. We can compute the two-point (as well as higher-point) correlation functions of the model through the unitarity sum (Figure 4)

$$
\begin{aligned}
& <\Phi(x) \Phi(0)>=\sum_{n=0}^{\infty} \int_{\theta_{1}>\theta_{2} \ldots>\theta_{n}} \frac{d \theta_{1}}{2 \pi} \cdots \frac{d \theta_{n}}{2 \pi} \\
& |<0| \Phi(0)\left|A_{a_{1}}\left(\theta_{1}\right) \cdots A_{a_{n}}\left(\theta_{n}\right)>\right|^{2} e^{-|x| \sum_{k=1}^{n} m_{k} \cosh \theta_{k}}
\end{aligned}
$$

Basic quantities of this approach are the Form Factors (FF), i.e. the matrix elements of the local operators $\Phi$ on the asymptotic states (Figure 5), defined as

$$
F_{a_{1}, \ldots, a_{n}}^{\Phi}\left(\theta_{1}, \ldots, \theta_{n}\right)=<0|\Phi(0)| A_{a_{1}}\left(\theta_{1}\right), \ldots, A_{a_{n}}\left(\theta_{n}\right)>.
$$

A detailed discussion of the form factor approach and the mathematical properties of the matrix elements (3.2) can be found in [6, 7]. Here we simply consider the basic equations we need for our discussion.

For a scalar operator $\Phi(x)$, relativistic invariance requires that its form factors depend only on the rapidity differences $\theta_{i}-\theta_{j}$. The elasticity of the scattering processes, together with the crossing symmetry and the completeness relation of the asymptotical states, permit to derive the following monodromy equations satisfied by the $\mathrm{FF}$

$$
\begin{aligned}
& F_{a_{1}, . ., a_{i}, a_{i+1}, . . a_{n}}^{\Phi}\left(\theta_{1}, . ., \theta_{i}, \theta_{i+1}, . ., \theta_{n}\right)= \\
& F_{a_{1}, a_{2}, \ldots a_{n}}^{\Phi}\left(\theta_{1}+2 \pi i, \ldots, \theta_{n-1}, \theta_{n}\right)=S_{a_{i} a_{i+1}}\left(\theta_{i}-\theta_{i+1}\right) F_{a_{1}, . a_{i+1}, a_{i}, . a_{n}}^{\Phi}\left(\theta_{1}, . ., \theta_{i+1}, \theta_{i}, . ., \theta_{n}\right) \\
& a_{2}, a_{3}, \ldots, a_{n}, a_{1} \\
& \left(\theta_{2}, \ldots, \theta_{n}, \theta_{1}\right) .
\end{aligned}
$$

In terms of the $s$-variable of the channel $\left(A_{a_{i}} A_{a_{j}}\right)$, the first equation in (3.3) implies that in the form factors there is a branch cut in the $s$-plane extending from $s=\left(m_{a_{i}}+m_{a_{j}}\right)^{2}$ to $s=\infty$. This is similar to what happens in the $S$-matrix. However, a difference between the analytic structure of the FF and the $S$-matrix comes from the second equation, which on the contrary shows that the FF do not have a $u$-channel cut extending from $s=-\infty$ to $s=\left(m_{a_{i}}-m_{a_{j}}\right)^{2}$.

Apart from these monodromy properties, the FF are expected to have poles induced by the singularities of the $S$-matrix. A particular role is played by the simple poles. Among them, we can select two different classes which admit a natural particle interpretation. The first type of simple poles are the so-called kinematical poles related to the annihilation processes of a pair of particle and anti-particle states. These singularities are located at $\theta_{a}-\theta_{\bar{a}}=i \pi$ and for the corresponding residue we have

$$
-i \lim _{\tilde{\theta} \rightarrow \theta}(\tilde{\theta}-\theta) F_{a, \bar{a}, a_{1}, \ldots, a_{n}}^{\Phi}\left(\tilde{\theta}+i \pi, \theta, \theta_{1}, \ldots, \theta_{n}\right)=\left(1-\prod_{j=1}^{n} S_{a, a_{j}}\left(\theta-\theta_{j}\right)\right) F_{a_{1}, \ldots, a_{n}}^{\Phi}\left(\theta_{1}, \ldots, \theta_{n}\right) .
$$


This equation can be graphically interpreted as an interference process due to the two different kinematical pictures drawn in Fig. 6.

A second class of simple poles is related to the presence of bound states appearing as simple poles in the $S$-matrix. If $\theta=i u_{a b}^{c}$ and $\Gamma_{a b}^{c}$ are the resonance angle and the three-particle coupling of the fusion $A_{a} \times A_{b} \rightarrow A_{c}$ respectively, then FF involving the particles $A_{a}$ and $A_{b}$ will also have a pole at $\theta=i u_{a b}^{c}$, with the residue given by (Fig. 7)

$$
-i \lim _{\theta_{a b} \rightarrow i u_{a b}^{c}}\left(\theta_{a b}-i u_{a b}^{c}\right) F_{a, b, a_{i}, \ldots, a_{n}}^{\Phi}\left(\theta_{a}, \theta_{b}, \theta_{1}, \ldots, \theta_{n}\right)=\Gamma_{a b}^{c} F_{c, a_{i}, \ldots, a_{n}}^{\Phi}\left(\theta_{c}, \theta_{1}, \ldots, \theta_{n}\right)
$$

where $\theta_{c}=\left(\theta_{a} \bar{u}_{b c}^{a}+\theta_{b} \bar{u}_{a c}^{b}\right) / u_{a b}^{c}$.

In general, the FF may also present simple poles which do not fall into the two classes above. In addition, they may also have higher-order poles and indeed, their analytic structure may be quite complicated. We will came back to this point in Section 4 where the specific example of the IMMF will be discussed.

Although eqs. (3.4) and (3.5) do not exhaust all pole information, nevertheless they induce a recursive structure in the space of the FF, which may be useful for their determinationf. Finding the general solution of eqs. (3.3), (3.4) and (3.5) for the IMMF poses a mathematical problem of formidable complexity, as described in Appendix A.

Fortunately, because of an important property of the spectral series (3.1), an accurate knowledge of the correlation functions can be reached with limited mathematical effort. This property consists of a very fast rate of convergence for all distance scales [8]. In view of this, the correlation functions can be determined with remarkable accuracy by truncating the series to the first few terms only. This statement appears to be obviously true in the infrared region (large $M r$ ), where more terms included into the series only add exponentially small contributions to the final result. The fast rate of convergence in the crossover and ultraviolet regions seems less obvious. In fact, for small values of the scaling variable $M r$, the correlators usually present power-law singularities and all numbers of particles are in principle supposed to significantly contribute to the sum. However, this does not seem to be the case of integrable QFT with sufficiently mild singularities in the ultraviolet region for a "threshold suppression effect" discussed in [8]. Although this result was originally derived for QFT with only one species of particles in the spectrum, we expect that it also applies to the IMMF the origin, i.e. $G(x) \sim x^{-1 / 4}$.

${ }^{4}$ An important general aspect of the Form Factor approach which is worth mentioning is that the validity of eqs. (3.3), (3.4) and (3.5) do not rely on the choice of any specific local operator $\Phi(x)$. This observation, originally presented in [10], may be used to classify the operator content of the massive integrable QFT, as explicitly shown in refs. 110, 29, 30, 31.

${ }^{5}$ As we will show in Sect. 5 , this will be indeed confirmed by: (a) a direct comparison with the numerical determination of the correlation function; (b) the saturation of the sum-rule related to the second derivative of the free-energy of the model (the zero-moment of the correlation function) and (c) 
If this crucial property of the spectral series is taken for granted, the first terms of the series are expected to saturate the values of the correlation function with a high degree of precision and we can only concentrate on their analytic determination. The number of terms to be included in the series essentially depends on the accuracy we would like to reach in the ultraviolet region and, to this aim, it is convenient to order them according to the energy of the particle states. For the IMMF, the first seventeen states are collected in Table 2. The most important contributions to the sum come from the one-particle states $A_{1}, A_{2}$ and $A_{3}$, and for the correlation function we have correspondingly

$G(r)=|<0| \sigma(0)|0>|^{2}+\frac{\left|\Upsilon_{1}\right|^{2}}{\pi} K_{0}\left(m_{1} r\right)+\frac{\left|\Upsilon_{2}\right|^{2}}{\pi} K_{0}\left(m_{2} r\right)+\frac{\left|\Upsilon_{3}\right|^{2}}{\pi} K_{0}\left(m_{3} r\right)+\mathcal{O}\left(e^{-2 m_{1} r}\right)$

where $K_{0}(x)$ is the modified Bessel function and we have defined

$$
\Upsilon_{i} \equiv<0|\sigma(0)| A_{i}>
$$

These matrix elements will be exactly determined in next section. Concerning the vacuum expectation value of $\sigma(x)$, it can be easily obtained from the relationship between this field and the trace $\Theta(x)$ of the stress-energy tensor. Since $\sigma(x)$ plays the role of the perturbing field in the theory under consideration, it is related to $\Theta(x)$ as

$$
\Theta(x)=2 \pi h\left(2-2 \Delta_{\sigma}\right) \sigma(x)
$$

On the other hand, the vacuum expectation of $\Theta(x)$ can be exactly determined by the Thermodynamic Bethe Ansatz and its value is given by [33]

$$
<0|\Theta(0)| 0>=\frac{\pi m_{1}^{2}}{\varphi_{11}}
$$

where

$$
\varphi_{11}=2 \sum_{\alpha \in \mathcal{A}_{11}} \sin \pi \alpha=2\left(\sin \frac{2 \pi}{3}+\sin \frac{2 \pi}{5}+\sin \frac{\pi}{15}\right) .
$$

Hence, using the above formulas and eq. (2.4), we have

$$
<0|\sigma(0)| 0>=\frac{4 \mathcal{C}^{2}}{15 \varphi_{11}} h^{1 / 15}=(1.07496 . .) h^{1 / 15}
$$

Eq. (3.6) provides the first terms of the large-distance expansion of the correlation function $<\sigma(x) \sigma(0)>$. A more refined determination of $G(x)$ may be obtained by computing the FF of the higher-mass states, as discussed in the next section and in Appendix A.

the saturation of the sum-rule derived from the $c$-theorem (second moment of the correlation function). 


\section{Form Factors of the IMMF}

In the framework of the Form Factor bootstrap approach to integrable theories, the twoparticle FF play a particularly important role, both from a theoretical and from a practical point of view. From a theoretical point of view, they provide the initial conditions which are needed for solving the recursive equations. Moreover, they also encode all the basic properties that the matrix elements with higher number of particles inherit by factorization, namely the asymptotic behaviour and the analytic structure. In other words, once the two-particle FF of the considered operator have been given, the determination of all other matrix elements is simply reduced to solve a well-defined mathematical problem. From a practical point of view, the truncation of the spectral series at the two-particle level usually provides a very accurate approximation of the correlation function, which goes even further than the crossover region. This section is mainly devoted to the discussion of the basic features of the two-particle FF in the IMMF.

In the general case, the $\mathrm{FF} F_{a b}^{\Phi}(\theta)$ must be a meromorphic function of the rapidity difference defined in the strip $\operatorname{Im} \theta \in(0,2 \pi)$. Its monodromy properties are dictated by the general equations (3.3), once specialized to the case $n=2$

$$
\begin{gathered}
F_{a b}^{\Phi}(\theta)=S_{a b}(\theta) F_{a b}^{\Phi}(-\theta), \\
F_{a b}^{\Phi}(i \pi+\theta)=F_{a b}^{\Phi}(i \pi-\theta) .
\end{gathered}
$$

Thus, denoting by $F_{a b}^{\min }(\theta)$ a solution of eqs. (4.1), (4.2) free of poles and zeros in the strip and also requiring asymptotic power boundness in momenta, we conclude that $F_{a b}^{\Phi}(\theta)$ must be equal to $F_{a b}^{\min }(\theta)$ times a rational function of $\cosh \theta$. The poles of this extra function are determined by the singularity structure of the scattering amplitude $S_{a b}(\theta)$.

A simple pole in $F_{a b}^{\Phi}(\theta)$ associated to the diagram of Fig. 8 corresponds to a positive residue simple pole in $S_{a b}(\theta)$ (see eq.(2.9)) and in this case we can write

$$
F_{a b}^{\Phi}\left(\theta \simeq i u_{a b}^{c}\right) \simeq \frac{i \Gamma_{a b}^{c}}{\theta-i u_{a b}^{c}} F_{c}^{\Phi}
$$

The single particle $\mathrm{FF} F_{c}^{\Phi}$ is a constant because of Lorentz invariance. Other poles induced by higher order singularities in the scattering amplitudes will be considered later in this section. The kinematical poles discussed in Section 3 do not appear at the two-particle level if the operator $\Phi(x)$ is local with respect to the fields which create the particles, which is the case of interest for us.

Summarizing, the two-particle FF can be parameterized as

$$
F_{a b}^{\Phi}(\theta)=\frac{Q_{a b}^{\Phi}(\theta)}{D_{a b}(\theta)} F_{a b}^{\min }(\theta),
$$


where $D_{a b}(\theta)$ and $Q_{a b}^{\Phi}(\theta)$ are polynomials in $\cosh \theta$ : the former is fixed by the singularity structure of $S_{a b}(\theta)$ while the latter carries the whole information about the operator $\Phi(x)$.

An upper bound on the asymptotic behaviour of FF and then on the order of the polynomial $Q_{a b}^{\Phi}(\theta)$ in eq. (4.4) comes from the following argument. Let $2 \Delta_{\Phi}$ be the scaling dimension of the scalar operator $\Phi(x)$ in the ultraviolet limit, i.e.

$$
<\Phi(x) \Phi(0)>\sim \frac{1}{|x|^{4 \Delta_{\Phi}}}, \quad|x| \rightarrow 0 .
$$

Then in a massive theory

$$
M_{p} \equiv \int d^{2} x|x|^{p}<\Phi(x) \Phi(0)>_{c} \quad<+\infty \quad \text { if } \quad p>4 \Delta_{\Phi}-2,
$$

where the subscript $c$ denotes the connected correlator. The two-point correlator may be expressed in terms of its euclidean Lehmann representation as

$$
<\Phi(x) \Phi(0)>_{c}=\int d^{2} p e^{i p x} \int d \mu^{2} \frac{\rho\left(\mu^{2}\right)}{p^{2}+\mu^{2}},
$$

where the spectral function $\rho$ is given by

$\rho\left(\mu^{2}\right)=\frac{1}{2 \pi} \sum_{n=1}^{\infty} \int_{\theta_{1}>\ldots>\theta_{n}} \frac{d \theta_{1}}{2 \pi} \ldots \frac{d \theta_{n}}{2 \pi}\left|F_{a_{1}, \ldots, a_{n}}^{\Phi}\left(\theta_{1}, \ldots, \theta_{n}\right)\right|^{2} \delta\left(\sum_{k=1}^{n} m_{k} \cosh \theta_{k}-\mu\right) \delta\left(\sum_{k=1}^{n} m_{k} \sinh \theta_{k}\right)$,

Substituting eq. (4.7) into the definition of $M_{p}$ and performing the integrations over $p, \mu$, and $x$, one finds

$$
M_{p} \sim \sum_{n=1}^{\infty} \int_{\theta_{1}>\ldots>\theta_{n}} d \theta_{1} \ldots d \theta_{n} \frac{\left|F_{a_{1}, \ldots, a_{n}}^{\Phi}\left(\theta_{1}, \ldots, \theta_{n}\right)\right|^{2}}{\left(\sum_{k=1}^{n} m_{k} \cosh \theta_{k}\right)^{p+1}} \delta\left(\sum_{k=1}^{n} m_{k} \sinh \theta_{k}\right) .
$$

Eq. (4.6) can now be used to derive an upper bound for the real quantity $y_{\Phi}$, defined by

$$
\lim _{\left|\theta_{i}\right| \rightarrow \infty} F_{a_{1}, \ldots, a_{n}}^{\Phi}\left(\theta_{1}, \ldots, \theta_{n}\right) \sim e^{y_{\Phi}\left|\theta_{i}\right|}
$$

This can be achieved by firstly noting that taking the limit $\theta_{i} \rightarrow+\infty$ in the integrand of eq. (4.8), the delta-function forces some other rapidity $\theta_{j}$ to diverge to minus infinity as $-\theta_{i}$. Since the matrix element $F_{a_{1}, \ldots, a_{n}}^{\Phi}\left(\theta_{1}, \ldots, \theta_{n}\right)$ depends on the rapidity differences, it will contribute to the integrand a factor $e^{2 y_{\Phi}\left|\theta_{i}\right|}$ in the limit $\left|\theta_{i}\right| \rightarrow \infty$. Then eq. (4.6) leads to the constraint

$$
y_{\Phi} \leq \Delta_{\Phi} .
$$

Note that this conclusion may not hold for non-unitary theories since not all the terms in the expansion over intermediate states are guaranteed to be positive in these cases. 
Let us see how the aforementioned considerations apply to the specific case of the IMMF. An appropriate solution of eqs. (4.1) and (4.2), corresponding to the scattering amplitudes reported in Table 1, can be written as

$$
F_{a b}^{\min }(\theta)=\left(-i \sinh \frac{\theta}{2}\right)^{\delta_{a b}} \prod_{\alpha \in \mathcal{A}_{a b}}\left(G_{\alpha}(\theta)\right)^{p_{\alpha}},
$$

where

$$
G_{\alpha}(\theta)=\exp \left\{2 \int_{0}^{\infty} \frac{d t}{t} \frac{\cosh \left(\alpha-\frac{1}{2}\right) t}{\cosh \frac{t}{2} \sinh t} \sin ^{2} \frac{(i \pi-\theta) t}{2 \pi}\right\} .
$$

For large values of the rapidity

$$
G_{\alpha}(\theta) \sim \exp (|\theta| / 2),|\theta| \rightarrow \infty,
$$

independent of the index $\alpha$. Other properties of this function are discussed in Appendix B.

An analysis of the two-particle FF singularities which will be described later on in this section, suggests that the pole terms appearing in the general parameterization eq. (4.4) could be written as

$$
D_{a b}(\theta)=\prod_{\alpha \in \mathcal{A}_{a b}}\left(\mathcal{P}_{\alpha}(\theta)\right)^{i_{\alpha}}\left(\mathcal{P}_{1-\alpha}(\theta)\right)^{j_{\alpha}}
$$

where

$$
\begin{array}{lll}
i_{\alpha}=n+1, & j_{\alpha}=n, & \text { if } \quad p_{\alpha}=2 n+1 ; \\
i_{\alpha}=n, & j_{\alpha}=n, & \text { if } \quad p_{\alpha}=2 n,
\end{array}
$$

and we have introduced the notation

$$
\mathcal{P}_{\alpha}(\theta) \equiv \frac{\cos \pi \alpha-\cosh \theta}{2 \cos ^{2} \frac{\pi \alpha}{2}} .
$$

Both $F_{a b}^{\min }(\theta)$ and $D_{a b}(\theta)$ have been normalized to 1 in $\theta=i \pi$.

Finally, let us turn our attention to the determination of the polynomials $Q_{a b}^{\Phi}(\theta)$ for the specific operator we are interested in, namely the magnetization field $\sigma(x)$. In view of the relation (3.8), this is the same as considering the analogous problem for the trace of the energy-momentum tensor $\Theta(x)$. For reasons which will become immediately clear, we will consider the latter operator in the remainder of this section.

The conservation equation $\partial_{\mu} T^{\mu \nu}=0$ implies the following relations among the $\mathrm{FF}$ of the different components of the energy-momentum tensor

$$
\begin{aligned}
& F_{a_{1}, \ldots, a_{n}}^{T^{++}}\left(\theta_{1}, \ldots, \theta_{n}\right) \sim \frac{P^{+}}{P_{-}} F_{a_{1}, \ldots, a_{n}}^{\Theta}\left(\theta_{1}, \ldots, \theta_{n}\right) ; \\
& F_{a_{1}, \ldots, a_{n}}^{T^{--}}\left(\theta_{1}, \ldots, \theta_{n}\right) \sim \frac{P^{-}}{P_{+}} F_{a_{1}, \ldots, a_{n}}^{\Theta}\left(\theta_{1}, \ldots, \theta_{n}\right),
\end{aligned}
$$


where $x^{ \pm}=x^{0} \pm x^{1}$ are the light-cone coordinates and $P^{ \pm} \equiv \sum_{i=1}^{n} p_{a_{i}}^{ \pm}$. The requirement that all the components of the energy-momentum tensor must exhibit the same singularity structure, leads to conclude that the FF of $\Theta(x)$ must contain a factor $P^{+} P^{-}$. However, the case $n=2$ is special because, if the two particles have equal masses, the mismatch of the singularities disappears in eqs. (4.18) and no factorisation takes place. From this analysis, we conclude that for our model we can write

$$
Q_{a b}^{\Theta}(\theta)=\left(\cosh \theta+\frac{m_{a}^{2}+m_{b}^{2}}{2 m_{a} m_{b}}\right)^{1-\delta_{a b}} P_{a b}(\theta)
$$

where

$$
P_{a b}(\theta) \equiv \sum_{k=0}^{N_{a b}} a_{a b}^{k} \cosh ^{k} \theta
$$

The degree $N_{a b}$ of the polynomials $P_{a b}(\theta)$ can be severely constrained by using eqs. (4.9) and (4.10). Additional conditions for these polynomials are provided by the normalization of the operator $\Theta(x)$, that for the diagonal elements $F_{a a}^{\Theta}$, reads

$$
F_{a a}^{\Theta}(i \pi)=<A_{a}\left(\theta_{a}\right)|\Theta(0)| A_{a}\left(\theta_{a}\right)>=2 \pi m_{a}^{2} .
$$

Using all the information above, we can now proceed in the computation of the IMMF form factors, starting from the simplest two-particle FF of the model, namely $F_{11}^{\Theta}(\theta)$ and $F_{12}^{\Theta}(\theta)$.

First of all, by using eqs. (4.10) and (4.13) one concludes that $N_{11} \leq 1$ and $N_{12} \leq 1$. In view of the normalization condition (4.21), only one unknown parameter, say $a_{11}^{1}$, is necessary in order to have the complete expression of $F_{11}^{\Theta}(\theta)$. On the contrary, we need two parameters, $a_{12}^{0}$ and $a_{12}^{1}$, to specify $F_{12}^{\Theta}(\theta)$. To determine all three unknown parameters, note that the scattering amplitude $S_{11}(\theta)$ possesses three positive residue poles at $\theta=i \frac{2 \pi}{3}$, $\theta=i \frac{2 \pi}{5}$ and $\theta=i \frac{\pi}{15}$ which correspond to the particles $A_{1}, A_{2}$ and $A_{3}$ respectively; on the other hand, $S_{12}(\theta)$ exhibits four positive residue poles at $\theta=i \frac{4 \pi}{5}, \theta=i \frac{3 \pi}{5}, \theta=i \frac{7 \pi}{15}$ and $\theta=i \frac{4 \pi}{15}$ associated to $A_{1}, A_{2}, A_{3}$ and $A_{4}$. Hence, since three poles are common to both amplitudes and no multiple poles appear in both of them, eq. (4.3) provides a system of three linear equations which uniquely determine the coefficients $a_{11}^{1}, a_{12}^{0}$ and $a_{12}^{1}$

$$
\frac{1}{\Gamma_{11}^{c}} \operatorname{Res}_{\theta=i u_{11}^{c}} F_{11}^{\Theta}(\theta)=\frac{1}{\Gamma_{12}^{c}} \operatorname{Res}_{\theta=i u_{12}^{c}} F_{12}^{\Theta}(\theta) \quad c=1,2,3 .
$$

The result of this calculation can be expressed in terms of the mass ratios $\hat{m}_{i}=m_{i} / m_{1}$ as

$$
\begin{aligned}
& P_{11}(\theta)=\frac{2 \pi m_{1}^{2}}{\hat{m}_{3} \hat{m}_{7}}\left(2 \cosh \theta+2+\hat{m}_{3} \hat{m}_{7}\right) \\
& P_{12}(\theta)=H_{12}\left(2 \hat{m}_{2} \cosh \theta+\hat{m}_{2}^{2}+\hat{m}_{8}^{2}\right)
\end{aligned}
$$


where

$$
H_{12}=(1.912618 . .) m_{1}^{2} .
$$

Equations (4.3) can now be used to obtain the one-particle form factors $F_{a}^{\Theta}(a=1, \ldots, 4)$, whose numerical values are reported in Table 3. In particular,

$$
\begin{gathered}
F_{1}^{\Theta}=\frac{\pi m_{1}^{2}}{\Gamma_{11}^{1} \hat{m}_{3} \hat{m}_{7}} \frac{\left(1+\hat{m}_{3} \hat{m}_{7}\right)\left[G_{\frac{2}{3}} G_{\frac{2}{5}} G_{\frac{1}{15}}\left(\frac{2 \pi i}{3}\right)\right] \cos ^{2} \frac{\pi}{5} \cos ^{2} \frac{\pi}{30}}{\sin \frac{8 \pi}{15} \sin \frac{2 \pi}{15} \sin \frac{3 \pi}{10} \sin \frac{11 \pi}{30}} \\
F_{2}^{\Theta}=-\frac{4 \pi m_{1}^{2}}{\Gamma_{11}^{2} \hat{m}_{3} \hat{m}_{7}} \frac{\sin \frac{\pi}{5}}{\sin \frac{2 \pi}{5}} \frac{\left(2 \cos \frac{2 \pi}{5}+2+\hat{m}_{3} \hat{m}_{7}\right)\left[G_{\frac{2}{3}} G_{\frac{2}{5}} G_{\frac{1}{15}}\left(\frac{2 \pi i}{5}\right)\right]\left(\cos \frac{\pi}{3} \cos \frac{\pi}{30} \cos \frac{\pi}{5}\right)^{2}}{\sin \frac{2 \pi}{5} \sin \frac{8 \pi}{15} \sin \frac{\pi}{6} \sin \frac{7 \pi}{30}} \\
F_{3}^{\Theta}=\frac{4 \pi m_{1}^{2}}{\Gamma_{11}^{3} \hat{m}_{3} \hat{m}_{7}} \frac{\sin \frac{\pi}{30}}{\sin \frac{\pi}{15}} \frac{\left(2 \cos \frac{\pi}{15}+2+\hat{m}_{3} \hat{m}_{7}\right)\left[G_{\frac{2}{3}} G_{\frac{2}{5}} G_{\frac{1}{15}}\left(\frac{i \pi}{15}\right)\right]\left(\cos \frac{\pi}{5} \cos \frac{\pi}{3} \cos ^{2} \frac{\pi}{30}\right)^{2}}{\sin \frac{3 \pi}{10} \sin \frac{11 \pi}{30} \sin \frac{\pi}{6} \sin \frac{7 \pi}{30}}
\end{gathered}
$$

In order to continue in the bootstrap procedure and compute the other one-particle and two-particle FF, we have to firstly consider the multiple poles of the scattering amplitudes. Such poles are known to represent the two-dimensional analog of anomalous thresholds associated to multi-scattering processes [18]. These are processes in which the two ingoing particles decay into their "constituents", which interact and then recombine to give a two-particle final state. In the general framework of relativistic scattering theory, the location of this kind of singularities is determined by the so-called Landau rules [21]. In the two-dimensional case, such rules admit the following simple formulation: singularities occur only for those values of the momenta for which a space-time diagram of the process can be drawn as a geometrical figure with all (internal and external) particles on mass-shell and energy-momentum conservation at the vertices. The simplified twodimensional kinematics only selects discrete values of the external momenta for which such a construction is possible and this is the reason why in two dimensions the "anomalous" singularities appear as poles rather than branch cuts. The order of the pole and its residue can be determined using the Cutkosky rule [21] which states that the discontinuity across the singularity associated to the abovementioned diagram is obtained evaluating it as if it were a Feynman graph but by inserting the complete scattering amplitudes at the interaction points and by replacing the internal propagators with mass-shell deltafunctions $\theta\left(p^{0}\right) \delta\left(p^{2}-m^{2}\right)$. For a diagram containing $P$ propagators and $L$ loops, $P-2 L$ delta-functions survive the $L$ two-dimensional integrations; since the singularity whose discontinuity is a single delta-function is a simple pole, the graph under consideration leads to a pole of order $P-2 L$ in the amplitude [19.

Let us initially consider the second order poles. A second order pole at $\theta=i \varphi$ occurs in the amplitude $S_{a b}(\theta)$ if one of the two diagrams in Figures 9.a and 9.b can be actually drawn, namely if

$$
\eta \equiv \pi-u_{c d}^{a}-u_{d e}^{b} \in[0, \pi)
$$


The quantity i $\eta$ is the rapidity difference between the intermediate propagating particles $A_{c}$ and $A_{e}$. From these figures, it is easy to see that

$$
\varphi=u_{a d}^{c}+u_{d b}^{e}-\pi
$$

The crossing symmetry expressed by eq. (2.7) obviously implies that, in addition to the double pole in $\theta=i \varphi$, an analogous pole must be present in $\theta=i(\pi-\varphi)$. Since the residues of the two poles are now equal, it is impossible to distinguish between a direct and a crossed channel, and the two poles must be treated on exactly the same footing. At the diagrammatic level, this fact is reflected by the possibility to find a diagram satisfying eq. (4.25) also for $\theta=i(\pi-\varphi)$. Hence, let us consider only one of these poles, the one located at $\theta=i \varphi$. In the vicinity of this pole, the scattering amplitude can be approximated as (see Fig. 9.a)

$$
S_{a b}(\theta) \simeq \frac{\left(\Gamma_{c d}^{a} \Gamma_{d e}^{b}\right)^{2} S_{c e}(\eta)}{(\theta-i \varphi)^{2}} .
$$

Note that the expression of this residue, which is obtained for $\eta>0$, is also valid in the limiting situation $\eta=0$ (Fig. 9.b), for which a residue $\left(\Gamma_{c d}^{a} \Gamma_{d e}^{b} \Gamma_{c f}^{b} \Gamma_{f e}^{a}\right)$ is expected. In fact, the consistency of the theory requires

$$
\Gamma_{c d}^{a} \Gamma_{d e}^{b}=\Gamma_{c f}^{b} \Gamma_{f e}^{a}
$$

an equation that is indeed satisfied for the three-point couplings of the IMMF. Moreover, in the case $\eta=0$, the "fermionic" nature of the particles, expressed by the relations

$$
S_{a b}(0)=\left\{\begin{array}{cll}
-1 & \text { if } & a=b \\
1 & \text { if } & a \neq b
\end{array}\right.
$$

implies that the two particles $A_{c}$ and $A_{e}$ propagating with the same momentum in Fig. 9.b cannot be of the same species. In this case, the factor $S_{c e}(\eta=0)$ in eq. (4.27) equals unity.

The double pole at $\theta=i \varphi$ in $S_{a b}(\theta)$ induces a singularity at the same position in $F_{a b}^{\Phi}(\theta)$. For $\eta>0$, this is associated to the diagram on the left hand side of Fig. 10. Since the singularity is now determined by a single triangular loop, the form factor $F_{a b}^{\Phi}(\theta)$ has only a simple pole rather than a double pole. The residue is given by

$$
\Gamma_{c d}^{a} \Gamma_{d e}^{b} S_{c e}(\eta) F_{c e}^{\Phi}(-\eta)
$$

Eq. (4.1) can now be used to write (see the right hand side of Fig. 10)

$$
F_{a b}^{\Phi}(\theta \simeq i \varphi) \simeq i \frac{\Gamma_{c d}^{a} \Gamma_{d e}^{b} F_{c e}^{\Phi}(\eta)}{\theta-i \varphi}
$$

As written, this result also holds for $\eta=0$. 
The poles of order $p>2$ in the scattering amplitudes and the corresponding singularities in the two-particle FF can be treated as a "composition" of the cases $p=1$ and $p=2$. This is the case, for instance, of a third order pole with positive residue at $\theta=i \varphi$ in $S_{a b}(\theta)$. In the $S$-matrix, a third order pole occurs if the scattering angle $\eta$ in Fig. 9.a coincides with the resonance angle $u_{c e}^{f}$. The corresponding diagram is drawn in Figure 11 and in this case we have

$$
S_{a b}(\theta \simeq i \varphi) \simeq i \frac{\left(\Gamma_{c d}^{a} \Gamma_{d e}^{b} \Gamma_{e c}^{f}\right)^{2}}{(\theta-i \varphi)^{3}} .
$$

The third-order pole at the crossing-symmetric position $\theta=i(\pi-\varphi)$ has negative residue since it corresponds to the crossed channel pole. With respect to the case $p=2$, the pole at $\theta=i \varphi$ in $F_{a b}^{\Phi}(\theta)$ becomes double (see Figure 12)

$$
F_{a b}^{\Phi}(\theta \simeq i \varphi) \simeq-\frac{\Gamma_{c d}^{a} \Gamma_{d e}^{b} \Gamma_{e c}^{f}}{(\theta-i \varphi)^{2}} F_{f}^{\Phi}
$$

while the pole at $\theta=i(\pi-\varphi)$ stays simple.

The above analysis suggests the validity of the following general pattern for the structure of the form-factor poles: a pole of order $2 n$ at $\theta=i \varphi$ in the crossing-symmetric scattering amplitude $S_{a b}(\theta)$ will induce a pole of order $n$ both at $\theta=i \varphi$ and at $\theta=i(\pi-\varphi)$ in the two-particle form factor $F_{a b}^{\Phi}(\theta)$; viceversa, a positive residue pole of order $(2 n+1)$ at $\theta=i \varphi$ in $S_{a b}(\theta)$ will induce a pole of order $(n+1)$ at $\theta=i \varphi$ and a pole of order $n$ at the crossing symmetrical position $\theta=i(\pi-\varphi)$ in $F_{a b}^{\Phi}(\theta)$. We have used this result to write the parameterization of eq. (4.14).

Moreover, in integrable QFT, these arguments can be easily extended to the higher matrix elements $F_{a_{1}, \ldots, a_{n}}^{\Phi}\left(\theta_{1}, \ldots, \theta_{n}\right)$ with $n>2$, since the complete factorisation of multiparticle processes prevents the generation of new singularities. In other words, the singularity structure of the n-particle FF is completely determined by the product of the poles present into each two-particle sub-channel.

We have used eqs. (4.3), (4.31), (4.33) to continue the bootstrap procedure for the two-particle $\mathrm{FF}$ of $\Theta(x)$ in the IMMF up to the level $A_{3} A_{3}$, and an illustration of the method through a specific example may be found in Appendix C. The results obtained for the coefficients $a_{a b}^{k}$ (the only unknown quantities in the parameterization of eq. (4.4) after the pole structure has been fixed) are summarized for convenience in Table 4; Table 3 contains the complete list of the one-particle matrix elements. Two important comments are in order here. The first is that in all the determinations of $F_{a b}^{\Theta}$ except $F_{11}^{\Theta}$ and $F_{12}^{\Theta}$, a number of equations larger than the number of unknown parameters is obtained. The

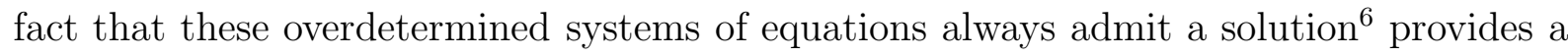

\footnotetext{
${ }^{6}$ Solutions can only be found by choosing the three-point couplings $\Gamma_{a b}^{c}$ either all positive or all negative. Hence, this restricts the ambiguity of the three-point couplings to an overall \pm sign only. We are not aware of any other explanation for this constraint on the $\Gamma_{a b}^{c}$.
} 
highly nontrivial check of the results of this section. The second point is that, since the pole structure has been identified, there is no obstacle, in principle, to continue further the bootstrap procedure and to achieve any desired precision in the determination of the correlation function in the ultraviolet region. Actually, we will show in the next section that the information contained in Tables 3 and 4 are more than enough for practical purposes. Nevertheless, from a purely theoretical point of view, it would be obviously desirable to have a complete solution of the recursive equations. A possible approach to this non trivial mathematical problem is suggested in Appendix A.

\section{Comparison with Numerical Simulations}

The data collected in Tables 3 and 4 , together with the vacuum expectation value eq. (3.9) and the three-particle matrix element $F_{111}^{\Theta}\left(\theta_{1}, \theta_{2}, \theta_{3}\right)$ given in Appendix A provide us with the complete large-distance expansion of the correlator $\langle\Theta(x) \Theta(0)>$ up to order $e^{-(m 2+m 3)|x|}$. A first check of the degree of convergence of the series, and then of its practical utility, is obtained by exploiting the exact knowledge of the second and zeroth moments of the correlation function we are considering. Indeed, in a massive theory the $c$-theorem sum rule provides the relation [32]

$$
C=\frac{3}{4 \pi} \int d^{2} x|x|^{2}<\Theta(x) \Theta(0)>_{c},
$$

where $C$ is the central charge of the conformal theory describing the ultraviolet fixed point. For the Ising model $C=\frac{1}{2}$. In addition, if we write the singular part of the free energy per unit volume as $f_{s} \simeq-U M^{2}(h)$, a double differentiation with respect to $h$ leads to the identity

$$
U=\frac{1}{\pi^{2}} \int d^{2} x<\Theta(x) \Theta(0)>_{c} .
$$

On the other hand, the exact value of the universal amplitude $U$ is obtained by plugging eq. (3.9) into

$$
U=\frac{4 \pi}{M^{2}(h)}<0|\Theta| 0>=0.0617286 . .
$$

The contributions to the sum rules (5.1) and (5.2) from the first eight states in the spectral representation of the connected correlator are listed in the Tables 5 and 6 , together with their partial sums. The numerical data are remarkably close to their theoretical values. Notice that a very fast saturation is also observed in the case of the zeroth moment, despite the absence of any suppression of the ultraviolet singularity.

Let us now directly compare the theoretical prediction of the connected correlation function $G_{c}(x)=<\sigma(x) \sigma(0)>_{c}$ with its numerical evaluation. A collection of highprecision numerical estimates of $G_{c}(x)$, for different values of the magnetic field $h$ and 
different size $L$ of the lattices, can be found in the reference [23]. We have decided to consider the set of data relative to $L=64$ and $h=0.075$, where the numerical values of $G(x)$ are known on 32 lattice space (Table 7). Such a choice was dictated purely by the requirement to use data where the effects of numerical errors are presumed to be minimized. Errors can be in fact induced either from the finite size $L$ of the sample or from the residue fluctuations of the critical point, which may be not sufficiently suppressed for small values of the magnetic field $h$.

In order to compare the numerical data with our theoretical determination, we only need to fix two quantities. The first consists in extracting the relationship between the inverse correlation length, expressed in lattice units, and the mass scale $M(h)$ entering the form factor expansion. The second quantity we need is the relative normalization of the operator $\sigma(x)$ defined on the lattice, denoted by $\sigma_{\text {lat }}(x)$, with the operator $\sigma(x)$ entering our theoretical calculation in the continuum limit. Let us consider the two issues separately.

The correlation length $\xi$ is easily extracted by using eq. (3.6) to analyse the exponential decay of the numerical data collected in Table 7 . As a best fit of this quantity, we obtain

$$
M(h=0.075)=\xi^{-1}=5.4(3) .
$$

Let us turn our attention to the second problem. The easiest way to set the normalization of $\sigma(x)$ with respect to $\sigma_{\text {lat }}(x)$ is to compare their vacuum expectation value. The lattice determination of this quantity can be found in [24, 23] and, within the numerical precision, it is given by

$$
<\sigma_{\text {lat }}(0)>=1.000(1) h^{1 / 15} .
$$

On the other hand, the theoretical estimate of $\langle\sigma(0)>$ was given in eq. (3.11). Hence, comparing the two results, the relative normalization is expressed by the constant $\mathcal{N}$ as

$$
\sigma_{\text {lat }}(x)=\mathcal{N} \sigma(x)=0.930(3) \sigma(x) .
$$

Once these two quantities are fixed, there are no more adjustable parameters to compare the numerical data with the large-distance expansion of $G_{c}(x)$. The form factors of the field $\sigma(x)$, entering the series (3.1) can be easily recovered from those of $\Theta(x)$, by using the relationship of these fields given by (3.8), and for the correlation function we have

$$
<\sigma(x) \sigma(0)>_{c}=\left(\frac{4}{15 \pi h}\right)^{2}<\Theta(x) \Theta(0)>_{c} .
$$

The comparison between the two determinations of $G_{c}(x)$ can be found in Figures 13 and 14. In Fig. 13 we have only included the first three terms of $G_{c}(x)$ (those relative to the form factors of the one-particle states $\left.A_{1}, A_{2}, A_{3}\right)$. As shown in this figure, they can 
reproduce correctly the behaviour of the correlation function on the whole infrared and crossover regions. A slight deviation of the theoretical curve from the numerical values is only observed for the first points of the ultraviolet region, where a better approximation can be obtained by including more terms in the form factor series. This is shown in Figure 14 , where five more contributions (those relative to form factors up to state $A_{1} A_{3}$ ) have been added to the series.

\section{Conclusion}

The basic results of this paper can be summarised as follows. The Zamolodchikov Smatrix for the IMMF has been used as the starting point to implement a bootstrap program for the FF of the magnetization operator. Although the general solution of the bootstrap recursive equations remains a challenging mathematical problem, the matrix elements yielding the main contributions to the spectral representation of the correlator $G(x)=<\sigma(x) \sigma(0)>$ have been explicitly computed. This has enabled us to write a large-distance expansion for $G(x)$ which is characterised by a very fast rate of convergence and provides accurate theoretical predictions for comparison with data coming from high precision numerical simulations.

It would be interesting to obtain analogous results for the other relevant operator of the theory, namely the energy density $\varepsilon(x)$. To this aim, the only difficulty one has to face is the determination of the initial conditions for the form factor bootstrap equations appropriate for this operator. In the case of the field $\sigma(x)$, we solved this problem by exploiting the proportionality with the trace $\Theta(x)$ of the energy-momentum tensor. Notice that, due to the absence of symmetries in the space of states of the IMMF, the occurrence of the polynomials $Q_{a b}^{\Phi}(\theta)$ in the two-particle $\mathrm{FF}$ is precisely what is needed in order to distinguish between the matrix elements of $\sigma(x)$ and those of $\varepsilon(x)$.

In conclusion, it must be remarked that the methods discussed in this paper can be generally used within the framework of integrable QFT. As a matter of fact, here they have been applied to a model which, for the absence of internal symmetries and the richness of its pole structure, can be considered as an extreme case of complexity. For instance, similar results to those contained in this paper can be obtained for other physically interesting situations, such as the thermal deformations of the tricritical Ising and three-state Potts models. The exact S-matrices for these models were determined in ref. 20, 35.

Acknowledgments. We are grateful to J.L. Cardy for useful discussions. 


\section{Appendix A}

Aim of this appendix is to formulate in general terms the mathematical problem related to the computation of a generic form factor of the scalar operators in the IMMF. Such a formulation is obtained by exploiting a decisive property of the model, namely its bootstrap structure: any particle $A_{i}(i=1,2, \ldots 8)$ of the theory appears as a bound state of some scattering process involving the fundamental particle $A_{1}$ and therefore can be obtained by a sufficient number of fusions of the particles $A_{1}$ 's alone. The simplest examples are provided by the particles $A_{2}$ and $A_{3}$, which appear in the initial amplitude $S_{11}(\theta)$. Hence, the bootstrap structure of the model implies that all possible FF of the theory can be in principle obtained by the $n$-particle FF which only involve the particle $A_{1}$, by simply applying the residue equations (3.5) the number of times we need to reach the FF under consideration. For instance, the form factors $F_{22}(\theta)$ and $F_{33}(\theta)$ can be both

obtained by starting from $F_{1111}$ and by applying twice (3.5) on the poles at $\frac{2 \pi i}{5}$ and $\frac{i \pi}{15}$, respectively.

In view of the role played by the $\mathrm{FF}$ with the particles $A_{1}$, it is convenient to use a convenient notation. For brevity, we will denote them as $F_{n}\left(\theta_{1}, \theta_{2}, \ldots, \theta_{n}\right) \equiv$ $F_{11 \ldots 1}\left(\theta_{1}, \ldots, \theta_{n}\right)$. It is now quite easy to find a parameterization of $F_{n}$ which correctly takes into account their monodromy properties and the pole structure. It can be written as

$$
F_{n}\left(\theta_{1}, \ldots, \theta_{n}\right)=H_{n} \frac{\Lambda_{n}\left(x_{1}, \ldots, x_{n}\right)}{\left(\omega_{n}\left(x_{1}, \ldots, x_{n}\right)\right)^{n}} \prod_{i<j} \frac{F_{11}^{\min }\left(\theta_{i j}\right)}{D_{11}\left(\theta_{i j}\right)\left(x_{i}+x_{j}\right)} .
$$

Let us explain the origin of each term entering the above equation.

The monodromy equations (3.3) can be satisfied in terms of the functions $F_{11}^{\min }(\theta)$, solution of the equations

$$
\begin{array}{ccc}
F_{11}^{\min }(\theta) & = & S_{11}(\theta) F_{11}^{\min }(-\theta) \\
F_{11}^{\min }(i \pi-\theta) & = & F_{11}^{\min }(i \pi+\theta)
\end{array}
$$

These functions are required to have neither zeros or poles in the strip $(0,2 \pi i) . F_{11}^{\min }(\theta)$ can be explicitly written in terms of the functions $G_{\lambda}(\theta)$ discussed in the appendix B as

$$
F_{11}^{\min }(\theta)=-i \sinh \frac{\theta}{2} G_{\frac{2}{3}}(\theta) G_{\frac{2}{5}}(\theta) G_{\frac{1}{15}}(\theta)
$$

Once the monodromy properties of $F_{n}$ are taken into account, we have to consider their pole structure. Note that, apart from the product of the $F_{11}^{\min }\left(\theta_{i j}\right)$ 's, the remaining part of these amplitudes can only be expressed in terms of functions of the variables $\theta_{i j}$ which are even and $2 \pi i$ periodic, i.e. functions of the variables $\cosh \theta_{i j}$. Equivalently, they have to be symmetric functions of the variables $x_{i} \equiv e^{\theta_{i}}$. A basis in the space of the 
symmetric functions of $n$-variables is provided by the elementary symmetric polynomials $\omega_{i}\left(x_{1}, x_{2}, \ldots, x_{n}\right)$ [34], defined by the generating function

$$
\prod_{k=1}^{n}\left(x+x_{i}\right)=\sum_{j=0}^{n} x^{n-j} \omega_{j}\left(x_{1}, \ldots, x_{n}\right)
$$

The bound state poles of $F_{n}$ in all possible subchannels of the amplitude $F_{n}$ is encoded in the product of the terms

$$
D_{11}(\theta) \equiv \mathcal{P}_{\frac{2}{3}}\left(\theta_{i j}\right) \mathcal{P}_{\frac{2}{5}}\left(\theta_{i j}\right) \mathcal{P}_{\frac{1}{15}}\left(\theta_{i j}\right)
$$

where $\mathcal{P}_{\lambda}(\theta)$ is defined in eq. (4.16). Concerning the kinematical poles, all of them are present in the product $\prod_{i<j}\left(x_{i}+x_{j}\right)$. Finally, in (A.1) $H_{n}$ is a normalization constant, $\Lambda_{n}\left(x_{1}, x_{2}, \ldots, x_{n}\right)$ is a symmetric polynomial and the last term $\left(\omega_{n}\left(x_{1}, \ldots, x_{n}\right)\right)^{n}$ (which has no zeros in the physical strip) has been inserted in order to have a convenient form of the recursive equations.

The polynomials $\Lambda_{n}\left(x_{1}, x_{2}, \ldots, x_{n}\right)$ can be obtained by solving the recursive equations (3.4) and (3.5). Using the parameterization (A.1), for the bound-state recursive equations we have

$$
\frac{\Lambda_{n+1}\left(e^{i \pi / 3} x, e^{-i \pi / 3} x, x_{2}, \ldots, x_{n}\right)}{x^{n+3}\left[\prod_{k=1}^{n}\left(x+x_{k}\right)\left(x-\eta x_{k}\right)\left(x-\eta^{-1} x_{k}\right)\right]}=\Lambda_{n}\left(x, x_{2}, \ldots, x_{n}\right),
$$

where $\eta=\exp (i \pi / 15)$. In writing (A.6) we have choose the normalization constants $H_{n}$ as

$$
H_{n+1}=\frac{\Gamma_{11}^{1} \sin \frac{2 \pi}{15} \sin \frac{11 \pi}{30} \sin \frac{8 \pi}{15} \sin \frac{3 \pi}{10}}{2 G_{11}\left(\frac{2 \pi i}{3}\right)\left(\cos \frac{\pi}{3} \cos \frac{\pi}{5} \cos \frac{\pi}{30}\right)^{2}}\left[\frac{\sin ^{2} \frac{11 \pi}{30}}{4 \gamma\left(\cos \frac{\pi}{3} \cos \frac{\pi}{5} \cos \frac{\pi}{30}\right)^{2}}\right]^{n} H_{n} .
$$

and, for simplicity, we have introduced the notation $G_{11}(\theta) \equiv G_{\frac{2}{3}}(\theta) G_{\frac{2}{5}}(\theta) G_{\frac{1}{15}}(\theta)$. The constant $\gamma$ is given by

$$
\gamma=\frac{G_{\frac{2}{3}}(0) G_{\frac{2}{3}}\left(\frac{2 \pi i}{3}\right) G_{\frac{2}{5}}\left(-\frac{i \pi}{3}\right) G_{\frac{2}{5}}\left(\frac{i \pi}{3}\right) G_{\frac{1}{15}}\left(-\frac{i \pi}{3}\right) G_{\frac{1}{15}}\left(\frac{i \pi}{3}\right)}{G_{1}\left(\frac{i \pi}{3}\right) G_{\frac{2}{3}}\left(\frac{i \pi}{3}\right) G_{\frac{11}{15}}(0) G_{\frac{1}{15}}(0) G_{\frac{2}{5}}(0) G_{-\frac{4}{15}}(0)} .
$$

A second hierarchy of recursive equations is obtained from the residue conditions of the kinematical poles, eq. (3.4), which can be written as

$$
(-)^{n} \Lambda_{n+2}\left(-x, x, x_{1}, \ldots, x_{n}\right)=\mathcal{A}_{n} U\left(x, x_{1}, \ldots, x_{n}\right) \Lambda_{n}\left(x_{1}, \ldots, x_{n}\right),
$$

where

$$
\mathcal{A}_{n}=\frac{\gamma G_{11}^{2}\left(\frac{2 \pi i}{3}\right)\left(2 \cos \frac{\pi}{3} \cos \frac{\pi}{5} \cos \frac{\pi}{30}\right)^{6}}{\sin ^{2} \frac{11 \pi}{30}\left(\Gamma_{11}^{1} \sin \frac{2 \pi}{15} \sin \frac{11 \pi}{30} \sin \frac{8 \pi}{15} \sin \frac{3 \pi}{10}\right)^{2}}\left[\frac{\gamma^{2} \sin \frac{2 \pi}{3} \sin \frac{2 \pi}{5} \sin \frac{\pi}{15}}{8 G_{11}(0) \sin ^{4} \frac{11 \pi}{30}}\right]^{n},
$$




$$
\begin{aligned}
& U\left(x, x_{1}, \ldots, x_{n}\right)=\frac{1}{2} x^{5} \sum_{k_{1}, \ldots, k_{6}=0}^{n}(-)^{k_{1}+k_{3}+k_{5}} \times \\
& x^{6 n-\left(k_{1}+\cdots k_{6}\right)} \sin \left[\frac{\pi}{15}\left(10\left(k_{1}-k_{2}\right)+6\left(k_{3}-k_{4}\right)+\left(k_{5}-k_{6}\right)\right)\right] \omega_{k_{1}} \ldots \omega_{k_{6}} .
\end{aligned}
$$

Note that for spinless operators, the total degree of the polynomials $\Lambda_{n}$ is equal to $\left(3 n^{2}-n\right) / 2$. The partial degree of $\Lambda_{n}$ in each variable $x_{i}$ is fixed by the asymptotic behaviour of the operator which is considered. For the FF of the spin operator $\sigma(x)$, the partial degree of $\Lambda_{n}$ is given by $(3 n-2)$.

Given the initial values of the recursive equations, namely the one-particle $\mathrm{FF} F_{1}$ and the two-particle FF $F_{2}$, one can solve eqs. (A.11) and (A.6) in order to get higher-particle FF. For instance, for the three-particle case we have

$$
\Lambda_{3}^{\Theta}\left(x_{1}, x_{2}, x_{3}\right)=-\mathcal{A}_{1} \omega_{1} \omega_{2}\left[A\left(\omega_{1} \omega_{2}^{4}+\omega_{1}^{3} \omega_{3}^{2}\right)+B \omega_{1}^{2} \omega_{2}^{2} \omega_{3}\right]
$$

where

$$
\begin{gathered}
A=\sin \frac{2 \pi}{3}+\sin \frac{\pi}{15}+\sin \frac{2 \pi}{5} \\
B=3 \sin \frac{2 \pi}{3}+2 \sin \frac{\pi}{15}+2 \sin \frac{2 \pi}{5}+\sin \frac{2 \pi}{15}-\sin \frac{\pi}{5}
\end{gathered}
$$

\section{Appendix B}

In this appendix we discuss some useful formulas for the calculation of the functions $F^{\min }(\theta)$ defined in the text. Let us consider a function $G_{\lambda}(\theta)$, solution of the equations

$$
\begin{array}{cl}
G_{\lambda}(\theta) & =f_{\lambda}(\theta) G_{\lambda}(-\theta) ; \\
G_{\lambda}(i \pi+\theta) & =G_{\lambda}(i \pi-\theta),
\end{array}
$$

where

$$
f_{\lambda}(\theta)=\frac{\tanh \frac{1}{2}(\theta+i \pi \lambda)}{\tanh \frac{1}{2}(\theta-i \pi \lambda)},
$$

without zeros and poles in the strip $(0,2 \pi i)$. This function admits several different representations. One of them is given by

$$
G_{\lambda}(\theta)=\exp \left[2 \int_{0}^{\infty} \frac{d t}{t} \frac{\cosh \left[\left(\lambda-\frac{1}{2}\right) t\right]}{\cosh \frac{t}{2} \sinh t} \sin ^{2} \frac{\hat{\theta} t}{2 \pi}\right]
$$

where $\hat{\theta} \equiv i \pi-\theta$. This function presents an infinite number of poles and zeros outside the strip $(0,2 \pi i)$, as shown by the following infinite-product representation

$$
G_{\lambda}(\theta)=\prod_{k=0}^{\infty}\left[\frac{\left[1+\left(\frac{\hat{\theta} / 2 \pi}{k+1-\frac{\lambda}{2}}\right)^{2}\right]\left[1+\left(\frac{\hat{\theta} / 2 \pi}{k+\frac{1}{2}+\frac{\lambda}{2}}\right)^{2}\right]}{\left[1+\left(\frac{\hat{\theta} / 2 \pi}{k+1+\frac{\lambda}{2}}\right)^{2}\right]\left[1+\left(\frac{\hat{\theta} / 2 \pi}{k+1-\frac{3}{2}-\frac{\lambda}{2}}\right)^{2}\right]}\right]^{k+1},
$$


or, equivalently

$$
G_{\lambda}(\theta)=\prod_{k=0}^{\infty}\left|\frac{\Gamma\left(\frac{1}{2}+k+\frac{\lambda}{2}\right) \Gamma\left(1+k-\frac{\lambda}{2}\right) \Gamma\left(1+k+\frac{\lambda}{2}+i \frac{\hat{\theta}}{2 \pi}\right) \Gamma\left(\frac{3}{2}+k-\frac{\lambda}{2}+i \frac{\hat{\theta}}{2 \pi}\right)}{\Gamma\left(\frac{3}{2}+k+\frac{\lambda}{2}\right) \Gamma\left(1+k+\frac{\lambda}{2}\right) \Gamma\left(1+k-\frac{\lambda}{2}+i \frac{\hat{\theta}}{2 \pi}\right) \Gamma\left(\frac{1}{2}+k+\frac{\lambda}{2}+i \frac{\hat{\theta}}{2 \pi}\right)}\right|^{2} .
$$

For numerical calculation, a quite useful expression is given by the mixed representation

$$
\begin{aligned}
& G_{\lambda}(\theta)=\prod_{k=0}^{N-1}\left[\frac{\left[1+\left(\frac{\hat{\theta} / 2 \pi}{k+1-\frac{\lambda}{2}}\right)^{2}\right]\left[1+\left(\frac{\hat{\theta} / 2 \pi}{k+\frac{1}{2}+\frac{\lambda}{2}}\right)^{2}\right]}{\left[1+\left(\frac{\hat{\theta} / 2 \pi}{k+1+\frac{\lambda}{2}}\right)^{2}\right]\left[1+\left(\frac{\hat{\theta} / 2 \pi}{k+1-\frac{3}{2}-\frac{\lambda}{2}}\right)^{2}\right]}\right]^{k+1} \times \\
& \exp \left[2 \int_{0}^{\infty} \frac{d t}{t} \frac{\cosh \left[\frac{t}{2}(1-2 \lambda)\right]}{\cosh \frac{t}{2} \sinh t}\left(N+1-N e^{-2 t}\right) e^{-2 N t} \sin ^{2} \frac{\hat{\theta} t}{2 \pi}\right] .
\end{aligned}
$$

From the above equations, we have

$$
G_{\lambda}(\theta)=G_{1-\lambda}(\theta)
$$

and

$$
G_{0}(\theta)=G_{1}(\theta)=-i \sinh \frac{\theta}{2} .
$$

The function $G_{\lambda}(\theta)$ satisfies the following functional equations

$$
\begin{gathered}
G_{\lambda}(\theta+i \pi) G_{\lambda}(\theta)=-i \frac{G_{\lambda}(0)}{\sin \pi \lambda}(\sinh \theta+i \sin \pi \lambda) \\
G_{\lambda}(\theta+i \pi \gamma) G_{\lambda}(\theta-i \pi \gamma)=\left(\frac{G_{\lambda}(i \pi \gamma) G_{\lambda}(-i \pi \gamma)}{G_{\lambda+\gamma}(0) G_{\lambda-\gamma}(0)}\right) G_{\lambda+\gamma}(\theta) G_{\lambda-\gamma}(\theta) \\
G_{1-\lambda}(\theta) G_{\lambda-1}(\theta)=\frac{\sinh \frac{1}{2}[\theta-i(\lambda-1) \pi] \sinh \frac{1}{2}[\theta+i(\lambda+1) \pi]}{\sin ^{2} \frac{\pi \lambda}{2}} .
\end{gathered}
$$

\section{Appendix C}

Aim of this Appendix is to illustrate through a specific example the procedure used to obtain the results listed in Tables 3 and 4 . Consider for instance the amplitude $S_{22}(\theta)$. It exhibits five simple positive residue poles located at $\theta=i \frac{4 \pi}{5}, \theta=i \frac{2 \pi}{3}, \theta=i \frac{7 \pi}{15}, \theta=i \frac{4 \pi}{15}$ and $\theta=i \frac{\pi}{15}$ which correspond to the bound states $A_{1}, A_{2}, A_{4}, A_{5}$ and $A_{6}$ respectively. In addition there is a pair of crossing-symmetric double poles at $\theta=i \frac{3 \pi}{5}$ and $\theta=i \frac{2 \pi}{5}$. The first one can be associated to the diagram of Figure 9. a with $a=b=2$ and $c=d=e=1$ while the second one can be associated to the diagram of Figure 9.b with $a=b=2$, $c=d=f=1$ and $e=2$. A simple way to check that these diagrams actually satisfy the required constraints (all the particles on mass-shell and energy-momentum conservation) 
is to draw the vector diagram for the momenta (dual diagram). Indeed, after performing the formal substitution $\theta_{k} \rightarrow i \beta_{k}$, any two-momentum $p_{k}^{\mu}=\left(m_{k} \cos \beta_{k}, i m_{k} \sin \beta_{k}\right)$ can be thought as a vector of length $m_{k}$ in the Euclidean plane. Then, the complete elasticity of the process requires that the external momenta form a closed parallelogram. Energymomentum conservation also implies that the momenta entering each three-particle vertex form closed triangles; as a simple consequence of eq.(2.8), the angle opposite to the side of length $m_{c}$ must be $\bar{u}_{a b}^{c} \equiv i \pi-u_{a b}^{c}$. If the triangles perfectly fit inside the external parallelogram, the original space-time diagram satisfy all the requirements. The dual diagrams associated to the double poles at $\theta=i \frac{3 \pi}{5}$ and $\theta=i \frac{2 \pi}{5}$ in the amplitude $S_{22}(\theta)$ are shown in Figures 15.a and 15.b respectively. Of course, the internal parallelogram in the first diagram corresponds to the central interaction in Figure 9.a.

It can be easily checked that

$$
\begin{gathered}
S_{22}\left(\theta \simeq \frac{3 i \pi}{5}\right) \simeq \frac{\left(\Gamma_{11}^{2}\right)^{4} S_{11}\left(\frac{i \pi}{5}\right)}{\left(\theta-\frac{3 i \pi}{5}\right)^{2}}, \\
\left(\Gamma_{11}^{2}\right)^{2} S_{11}\left(\frac{i \pi}{5}\right)=\left(\Gamma_{22}^{1}\right)^{2} .
\end{gathered}
$$

According to the general discussion of Section 4, we write the $\mathrm{FF} F_{22}^{\Theta}(\theta)$ as

$$
F_{22}^{\Theta}(\theta)=Q_{22}^{\Theta}(\theta) \frac{F_{22}^{\min }(\theta)}{D_{22}(\theta)},
$$

where

$$
\begin{gathered}
F_{22}^{\min }(\theta)=-i \sinh \frac{\theta}{2}\left[G_{\frac{4}{5}} G_{\frac{2}{3}} G_{\frac{7}{15}} G_{\frac{4}{15}} G_{\frac{1}{15}} G_{\frac{3}{5}}^{2}\right](\theta), \\
D_{22}(\theta)=\left[P_{\frac{4}{5}} P_{\frac{2}{3}} P_{\frac{7}{15}} P_{\frac{4}{15}} P_{\frac{1}{15}} P_{\frac{3}{5}} P_{\frac{2}{5}}\right](\theta) \\
Q_{22}^{\Theta}(\theta)=\sum_{k=0}^{N_{22}} a_{22}^{k} \cosh ^{k} \theta
\end{gathered}
$$

Notice that

$$
\begin{aligned}
F_{22}^{\min }(\theta) & \sim e^{4|\theta|}, & & |\theta| \rightarrow \infty, \\
D_{22}(\theta) & \sim e^{7|\theta|}, & & |\theta| \rightarrow \infty .
\end{aligned}
$$

In view of these asymptotic behaviours, eqs. (4.9), (4.10) imply that $N_{22} \leq 3$. Since $F_{11}^{\Theta}, F_{12}^{\Theta}(\theta)$ and $F_{a}^{\Theta}, a=1, \ldots, 5$ have been already determined in previous steps of the bootstrap procedure, eqs. (4.21), (4.3) and (4.31) provide the following seven linear equations for the four unknowns $a_{22}^{k}, k=0, \ldots, 3$

$$
\begin{gathered}
F_{22}^{\Theta}(i \pi)=2 \pi m_{2}^{2}, \\
F_{22}^{\Theta}\left(\theta \simeq i u_{22}^{c}\right) \simeq i \frac{\Gamma_{22}^{c}}{\theta-i u_{22}^{c}} F_{c}^{\Theta}, \quad c=1,2,4,5,
\end{gathered}
$$




$$
\begin{gathered}
F_{22}^{\Theta}\left(\theta \simeq \frac{3 i \pi}{5}\right) \simeq i \frac{\left(\Gamma_{11}^{2}\right)^{2}}{\theta-\frac{3 i \pi}{5}} F_{11}^{\Theta}\left(\frac{i \pi}{5}\right) \\
F_{22}^{\Theta}\left(\theta \simeq \frac{2 i \pi}{5}\right) \simeq i \frac{\Gamma_{11}^{2} \Gamma_{12}^{2}}{\theta-\frac{2 i \pi}{5}} F_{12}^{\Theta}(0)
\end{gathered}
$$

These equations turn out to be compatible and the solution is contained in Table 4 . The particle $A_{6}$ appears for the first time as a bound state in the amplitude $S_{22}(\theta)$. Since $F_{22}^{\Theta}(\theta)$ has been fixed, $F_{6}^{\Theta}$ can now be extracted using again eq. (4.3). 


\section{References}

[1] C. Itzykson, H. Saleur and J.B. Zuber, Conformal Invariance and Applications to Statistical Mechanics, (World Scientific, Singapore 1988) and references therein.

[2] G. Mussardo, Phys. Reports 218 (1992), 215 and references therein.

[3] A.A. Belavin, A.M. Polyakov and A.B. Zamolodchikov, Nucl. Phys. B 241 (1984), 333.

[4] A.B. Zamolodchikov, in Advanced Studies in Pure Mathematics 19 (1989), 641; Int. J. Mod. Phys. A 3 (1988), 743.

[5] A.B. Zamolodchikov, Al.B. Zamolodchikov, Ann.Phys. 120 (1979), 253.

[6] B. Berg, M. Karowski, P. Weisz, Phys. Rev. D 19 (1979), 2477; M. Karowski, P. Weisz, Nucl. Phys. B 139 (1978), 445; M. Karowski, Phys. Rep. 49 (1979), 229;

[7] F.A. Smirnov, Form Factors in Completely Integrable Models of Quantum Field Theory (World Scientific) 1992, and references therein.

[8] J.L. Cardy and G. Mussardo, Nucl. Phys. B 410 [FS] (1993), 451.

[9] V.P. Yurov and Al.B. Zamolodchikov, Int. J. Mod. Phys. A 6 (1991), 3419.

[10] J.L. Cardy and G. Mussardo, Nucl. Phys. B 340 (1990), 387.

[11] Al.B. Zamolodchikov, Nucl. Phys. B 348 (1991), 619.

[12] G. Delfino and G. Mussardo, Phys. Lett. B 324 (1994), 40; J. Balog, Phys. Lett. B 300 (1993), 145.

[13] A. Fring, G. Mussardo and P. Simonetti, Nucl. Phys. B 393 (1993), 413; G. Mussardo and P. Simonetti, Int. J. Mod. Phys. A 9 (1994), 3307.

[14] T.T. Wu, B.M. McCoy, C.A. Tracy and E. Barouch, Phys. Rev. B 13 (1978), 316.

[15] B.M. McCoy and T.T. Wu, The Two-Dimensional Ising Model (Harvard University Press, Cambridge, 1973).

[16] B.M. McCoy C.A. Tracy and T.T. Wu, Jour. Math. Phys. 18 (1977), 1058.

[17] O. Babelon and D. Bernard, Phys. Lett. B 288 (1992), 113.

[18] S. Coleman and H.J. Thun, Commun. Math. Phys. 61 (1978), 31; C.J. Goebel, Prog. Theor. Phys. Supplement 86 (1986), 261. 
[19] H.W. Braden, E. Corrigan, P.E. Dorey and R. Sasaki, Nucl. Phys. B 338 (1990), 689; Nucl. Phys. B 356 (1991), 469.

[20] P. Christe and G. Mussardo, Nucl. Phys. B 330 (1990), 465; Int. J. Mod. Phys. A 5 (1990), 4581.

[21] R.J. Eden, P.V. Landshoff, D.I. Olive and J.C. Polkinghorne, The Analytic S-matrix (Cambridge University Press) 1966.

[22] P.G. Lauwers and V. Rittenberg, Phys. Lett. B 233 (1989), 197.

[23] P.G. Lauwers and V. Rittenberg, Numerical Estimates of the Spin-Spin Correlation Function for the Critical 2-D Ising Model in a Magnetic Field, Bonn-HE-89-11.

[24] C. Destri, F. Di Renzo, E. Onofri, P. Rossi and G.P. Tecchiolli, Phys. Lett. B 278 (1992), 311.

[25] V.P. Yurov and Al.B. Zamolodchikov, Int. J. Mod. Phys. A 6 (1991), 4557; M. Henkel and H. Saleur, J. Phys. A 22 L513 (1989); I.R. Sagdeev and A.B. Zamolodchikov, Mod. Phys. Lett. B 3 (1989), 1375.

[26] V.V. Bazhanov, B. Nienhuis, and S.O. Warnaar, Phys. Lett. B 322 (1994), 198.

[27] B.M. McCoy and T.T. Wu, Phys. Rev. D 18 (1978), 1259; B.M. McCoy and M.L. Yan, Nucl. Phys. B 257 [FS14] (1985), 303.

[28] Vl.S. Dotsenko, Nucl. Phys. B 314 (1989), 687; Int. J. Mod. Phys. B 4 (1990), 1039.

[29] A. Koubek and G. Mussardo, Phys. Lett. B 311 (1993), 193.

[30] A. Koubek, Phys. Lett. B 346 (1995), 275; Nucl. Phys. B 428 (1994), 655; Nucl. Phys. B 435 (1995), 703.

[31] F.A. Smirnov, Counting the local fields in Sine-Gordon theory, hep-th 9501059.

[32] A.B. Zamolodchikov, JETP Lett. 43 (1986), 730; J.L. Cardy, Phys. Rev. Lett. 60 (1988), 2709.

[33] V.A. Fateev, Phys. Lett. B 324 (1994), 45.

[34] I.G. MacDonald, Symmetric functions and Hall polynomials (Claredon Press, Oxford, 1979).

[35] V.A. Fateev and A.B. Zamolodchikov, Int. Journ. of Mod. Phys. A 5 (1990), 1025. 


\section{Table Caption}

Table 1 . S-matrix elements of the IMMF where the numbers $(\gamma)$ in parenthesis should

be read in units of $\frac{1}{30}$. They represent the functions $\tanh \frac{1}{2}\left(\theta+i \pi \frac{\gamma}{30}\right) / \tanh \frac{1}{2}\left(\theta-i \pi \frac{\gamma}{30}\right)$. The bound state poles related to the particles $A_{i}(i=1, \ldots, 8)$ are identified by the numbers $i$ placed above the functions $(\gamma)$.

Table 2 . The first seventeen lowest energy states entering the spectral series.

Table 3 . One-particle Form Factors of the operator $\Theta$.

Table 4 . Coefficients of the polynomials $P_{a b}(\theta)$.

Table 5 . The first eight contributions to the $c$-theorem.

Table 6 . The first eight contributions to the universal amplitude of the free-energy.

Table 7 . Numerical values of $G_{c}(x)$ and their errors $\delta$ for $L=64$ and $h=0.075$, as determined in [23]. 


\section{Figure Caption}

Figure 1 . Two-body $S$-matrix.

Figure 2 . Analytic structure of the $S$-matrix in the $s$ plane. The circles indicate the location of the bound state poles.

Figure 3 . Bound state diagram in the $s$-channel.

Figure 4 . Spectral representation series of the correlator.

Figure 5 . Form Factor of the operator $\Phi$.

Figure 6 . The two kinematical situations (a) and (b) entering the residue equations of the annihilation poles.

Figure 7 . Bound state bootstrap equation for the Form Factor.

Figure 8 . Residue equation for the bound state poles of the two-particle Form Factor.

Figure 9 . Multi-scattering processes resulting in the double poles of the $S$-matrix.

Figure 10 . Residue equation for the simple poles of the Form Factor associated to the double poles of the $S$-matrix.

Figure 11 . Third-order pole in the $S$-matrix.

Figure 12 . Residue equation for the double poles of the Form Factor associated to the third-order poles of the $S$-matrix.

Figure 13 . Correlation function $\left\langle\sigma(x) \sigma(0)>_{c}\right.$ versus lattice space distances. The points on the graph represent the numerical data, as extracted from Table 7, while the continuum curve is the theoretical estimate with only the first three Form Factors.

Figure 14. Correlation function $\left\langle\sigma(x) \sigma(0)>_{c}\right.$ versus lattice space distances. The points on the graph represent the numerical data, as extracted from Table 7 while the continuum curve is the theoretical estimate obtained with the first eight terms of the spectral series.

Figure 15 . Dual diagrams of double pole graphs. 


\begin{tabular}{|c|c|}
\hline$a$ & $S_{a b}$ \\
\hline & $\begin{array}{ccc}\mathbf{1} & \mathbf{2} & \mathbf{3} \\
(20) & (12) & (2)\end{array}$ \\
\hline 1 & $\begin{array}{cccc}\mathbf{1} & \mathbf{2} & \mathbf{3} & \mathbf{4} \\
(24) & (18) & (14) & (8)\end{array}$ \\
\hline & $\begin{array}{cccc}\mathbf{1} & \mathbf{2} & \mathbf{4} & \mathbf{5} \\
(29) & (21)(13)(3) & (11)^{2}\end{array}$ \\
\hline & 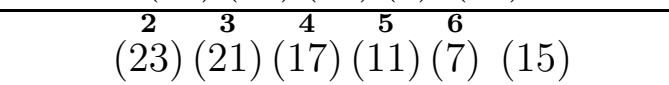 \\
\hline & 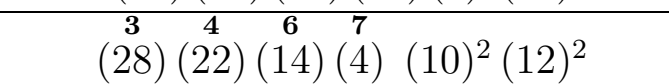 \\
\hline & 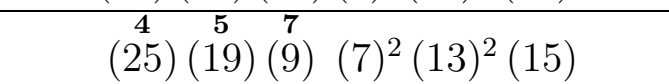 \\
\hline & 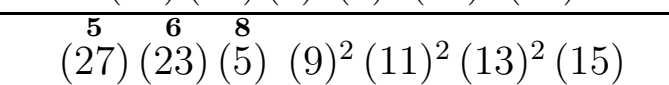 \\
\hline & $(26)(16)^{3}(6)^{2}(8)^{2}(10)^{2}(12)^{2}$ \\
\hline & 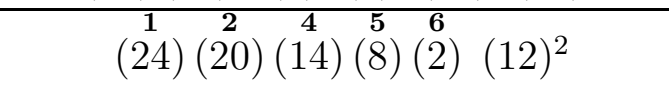 \\
\hline 2 & $\begin{array}{ccc}\mathbf{1} & \mathbf{3} & \mathbf{6} \\
(25) & (19) & (9) \\
(7)^{2}(13)^{2}(15)\end{array}$ \\
\hline 2 & 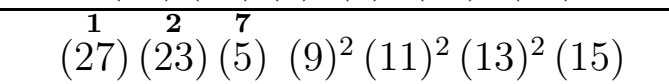 \\
\hline 2 & $\begin{array}{c}\mathbf{2} \\
(26)(16)^{3}\end{array}(6)^{2}(8)^{2}(10)^{2}(12)^{2}$ \\
\hline 2 & $\begin{array}{ccccc}\mathbf{2} & \mathbf{3} & \mathbf{5} & \mathbf{7} & \mathbf{8} \\
(29) & (25) & (19)^{3} & (13)^{3}(3) & (7)^{2}(9)^{2}(15)\end{array}$ \\
\hline 2 & $\begin{array}{cccc}\mathbf{4} & \mathbf{6} & \mathbf{7} & \mathbf{8} \\
(27)(21)^{3} & (17)^{3} & (11)^{3} & (5)^{2}(7)^{2}(15)^{2}\end{array}$ \\
\hline & $\begin{array}{cc}\mathbf{6} & \mathbf{7} \\
(28) & (22)^{3}(4)^{2}(6)^{2}(10)^{4}(12)^{4}(16)^{4}\end{array}$ \\
\hline 3 & $\begin{array}{ccccc}\mathbf{2} & \mathbf{3} & \mathbf{5} & \mathbf{6} & \mathbf{7} \\
(22)(20)^{3} & (14)(12)^{3}(4) & (2)^{2}\end{array}$ \\
\hline 3 & $\begin{array}{c}\mathbf{1} \\
(26) \\
(16)^{3}\end{array}(6)^{2}(8)^{2}(10)^{2}(12)^{2}$ \\
\hline 3 & $\begin{array}{ccccc}\mathbf{1} & \mathbf{3} & \mathbf{4} & \mathbf{7} & \mathbf{8} \\
(29)(23)(21)^{3} & (13)^{3}(5)(3)^{2}(11)^{4}(15)\end{array}$ \\
\hline & $\begin{array}{ccccc}\mathbf{2} & \mathbf{3} & \mathbf{6} & \mathbf{8} & \\
(26) & (24)^{3} & (18)^{3} & (8)^{3} & (10)^{2}(16)^{4}\end{array}$ \\
\hline 3 & $\begin{array}{ccc}\mathbf{3} & \mathbf{5} \\
(28) & (22)^{3} & (4)^{2}(6)^{2}(10)^{4}(12)^{4}(16)^{4}\end{array}$ \\
\hline 3 & $\begin{array}{cccc}\mathbf{5} & \mathbf{6} & \mathbf{8} & \\
(27) & (25)^{3} & (17)^{5} & (7)^{4}(9)^{4}(11)^{2}(15)^{3}\end{array}$ \\
\hline
\end{tabular}

Continued 


\begin{tabular}{|c|c|}
\hline$a$ & $S_{a b}$ \\
\hline 4 & $\begin{array}{cccccc}\mathbf{1} & \mathbf{4} & \mathbf{6} & \mathbf{7} & \mathbf{8} & \\
(26) & (20)^{3} & (16)^{3} & (12)^{3} & (2) & (6)^{2}(8)^{2}\end{array}$ \\
\hline 4 & $\begin{array}{cccc}\mathbf{1} & \mathbf{3} & \mathbf{5} & \mathbf{8} \\
(27)(23)^{3} & (19)^{3} & (9)^{3} & (5)^{2}(13)^{4}(15)^{2}\end{array}$ \\
\hline 4 & $\begin{array}{cc}\mathbf{1} \\
(28) & \mathbf{4} \\
(22)^{3} & (4)^{2}(6)^{2}(10)^{4}(12)^{4}(16)^{4}\end{array}$ \\
\hline & $\begin{array}{ccccc}\mathbf{2} & \mathbf{4} & \mathbf{7} & \mathbf{8} & \\
(28) & (24)^{3} & (18)^{5} & (14)^{5} & (4)^{2}(8)^{4}(10)^{4}\end{array}$ \\
\hline & 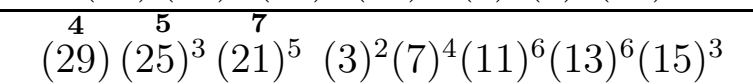 \\
\hline & $\begin{array}{ccc}\mathbf{4} & \mathbf{5} & \mathbf{8} \\
(22)^{3} & (20)^{5} & (12)^{5} \\
(2)^{2}(4)^{2}(6)^{2}(16)^{4}\end{array}$ \\
\hline & $\left.\begin{array}{ccc}\mathbf{1} & \mathbf{2} \\
(27) & (25)^{3} & (17 \\
\end{array}\right)^{5}(7)^{4}(9)^{4}(11)^{4}(15)^{3}$ \\
\hline 5 & $(29)(25)^{3}(21)^{5}(3)^{2}(7)^{4}(11)^{6}(13)^{6}(15)^{3}$ \\
\hline 5 & $\begin{array}{ccccc}\mathbf{3} & \mathbf{4} & \mathbf{5} & \mathbf{8} & \\
(28) & (26)^{3} & (24)^{5} & (18)^{7} & (8)^{6} \\
(10)^{6} & (16)^{8}\end{array}$ \\
\hline 6 & $\begin{array}{cccc}\mathbf{3} & \mathbf{6} & \mathbf{8} \\
(24)^{3} & (20)^{5} & (14)^{5} & (2)^{2}(4)^{2}(8)^{4}(12)^{6}\end{array}$ \\
\hline 6 & $\begin{array}{ccccc}\mathbf{1} & \mathbf{2} & \mathbf{5} & \mathbf{8} & \\
(28) & (26)^{3} & (22)^{5} & (16)^{7} & (6)^{4}(10)^{6}(12)^{6}\end{array}$ \\
\hline 6 & 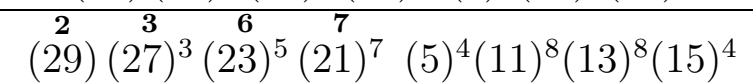 \\
\hline 7 & 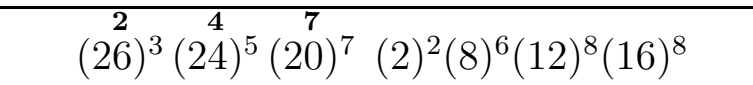 \\
\hline 7 & $\begin{array}{cccccc}\mathbf{1} & \mathbf{2} & \mathbf{4} & \mathbf{6} & \mathbf{8} & \\
(29) & (27)^{3} & (25)^{5} & (23)^{7} & (19)^{9} & (9)^{8}(13)^{10}(15)^{5}\end{array}$ \\
\hline 8 & $(28)^{3}(26)^{5}(24)^{7}(22)^{9}(20)^{11}(12)^{12}(16)^{12}$ \\
\hline
\end{tabular}

Table 1 


\begin{tabular}{|c|c|}
\hline $\mid n>$ & $\sqrt{s}$ \\
\hline \hline$A_{1}$ & $m_{1}$ \\
\hline$A_{2}$ & $(1.6180 ..) m_{1}$ \\
\hline$A_{3}$ & $(1.9890 ..) m_{1}$ \\
\hline$A_{1} A_{1}$ & $\geq 2 m_{1}$ \\
\hline$A_{4}$ & $(2.4048 ..) m_{1}$ \\
\hline$A_{1} A_{2}$ & $\geq(2.6180 ..) m_{1}$ \\
\hline$A_{5}$ & $(2.9563 ..) m_{1}$ \\
\hline$A_{1} A_{3}$ & $\geq(2.9890 ..) m_{1}$ \\
\hline$A_{1} A_{1} A_{1}$ & $\geq 3 m_{1}$ \\
\hline$A_{6}$ & $(3.2183 ..) m_{1}$ \\
\hline$A_{2} A_{2}$ & $\geq(3.2360 ..) m_{1}$ \\
\hline$A_{1} A_{4}$ & $\geq(3.4048 ..) m_{1}$ \\
\hline$A_{2} A_{3}$ & $\geq(3.6070 ..) m_{1}$ \\
\hline$A_{1} A_{1} A_{2}$ & $\geq(3.6180 ..) m_{1}$ \\
\hline$A_{7}$ & $3.8911 . . m_{1}$ \\
\hline$A_{1} A_{5}$ & $\geq(3.9563 ..) m_{1}$ \\
\hline$A_{3} A_{3}$ & $\geq(3.9780 ..) m_{1}$ \\
\hline
\end{tabular}

Table 2 


\begin{tabular}{|c|r|}
\hline$F_{1}^{\Theta}$ & 0.4971505471 \\
$F_{2}^{\Theta}$ & -0.2627111760 \\
$F_{3}^{\Theta}$ & 0.1447685755 \\
$F_{4}^{\Theta}$ & -0.1107486745 \\
$F_{5}^{\Theta}$ & -0.0467951944 \\
$F_{6}^{\Theta}$ & 0.0336573286 \\
$F_{7}^{\Theta}$ & -0.0127414814 \\
$F_{8}^{\Theta}$ & 0.0023550931 \\
\hline
\end{tabular}

Table 3 


\begin{tabular}{|l|}
\hline$a_{11}^{1}=1.623628945$ \\
$a_{11}^{0}=7.906814252$ \\
\hline$a_{12}^{1}=6.189362113$ \\
$a_{12}^{0}=48.76949783$ \\
\hline$a_{13}^{2}=451.6582994$ \\
$a_{13}^{1}=4824.093743$ \\
$a_{13}^{0}=4389.138083$ \\
\hline$a_{22}^{3}=16.66647213$ \\
$a_{22}^{2}=258.9398602$ \\
$a_{22}^{1}=613.8726746$ \\
$a_{22}^{0}=388.0488792$ \\
\hline$a_{14}^{2}=-17.51368456$ \\
$a_{14}^{1}=-222.5261059$ \\
$a_{14}^{0}=-207.6061104$ \\
\hline$a_{23}^{3}=71.93457181$ \\
$a_{23}^{2}=1358.926475$ \\
$a_{23}^{1}=3745.854658$ \\
$a_{23}^{0}=2718.541527$ \\
\hline$a_{15}^{3}=202.2760111$ \\
$a_{15}^{2}=3328.024309$ \\
$a_{15}^{1}=6286.863589$ \\
$a_{15}^{0}=3162.939632$ \\
\hline$a_{33}^{5}=928.5620526$ \\
$a_{33}^{4}=23400.66614$ \\
$a_{33}^{3}=116753.1311$ \\
$a_{33}^{2}=233559.8778$ \\
$a_{33}^{1}=207377.6722$ \\
$a_{33}^{0}=68123.67968$ \\
\hline
\end{tabular}

Table 4 


\begin{tabular}{|l|l|}
\hline$C_{1}$ & 0.472038282 \\
$C_{2}$ & 0.019231268 \\
$C_{3}$ & 0.002557246 \\
$C_{11}$ & 0.003919717 \\
$C_{4}$ & 0.000700348 \\
$C_{12}$ & 0.000974265 \\
$C_{5}$ & 0.000054754 \\
$C_{13}$ & 0.000154186 \\
\hline$C_{\text {partial }}$ & 0.499630066 \\
\hline
\end{tabular}

Table 5 


\begin{tabular}{|l|l|}
\hline$U_{1}$ & 0.050084817 \\
$U_{2}$ & 0.005342101 \\
$U_{3}$ & 0.001073471 \\
$U_{11}$ & 0.002496286 \\
$U_{4}$ & 0.000429759 \\
$U_{12}$ & 0.001031748 \\
$U_{5}$ & 0.000050773 \\
$U_{13}$ & 0.000225505 \\
\hline$U_{\text {partial }}$ & 0.060734461 \\
\hline
\end{tabular}

Table 6 


\begin{tabular}{|c|c|c|}
\hline$x$ & $G(x)$ & $\delta$ \\
\hline \hline 1 & 0.210791 & 0.000045 \\
\hline 2 & 0.118830 & 0.000035 \\
\hline 3 & 0.076439 & 0.000029 \\
\hline 4 & 0.052763 & 0.000024 \\
\hline 5 & 0.037944 & 0.000021 \\
\hline 6 & 0.028009 & 0.000018 \\
\hline 7 & 0.021060 & 0.000016 \\
\hline 8 & 0.016049 & 0.000013 \\
\hline 9 & 0.012356 & 0.000012 \\
\hline 10 & 0.009587 & 0.000011 \\
\hline 11 & 0.007493 & 0.000010 \\
\hline 12 & 0.005887 & 0.000008 \\
\hline 13 & 0.004645 & 0.000009 \\
\hline 14 & 0.003683 & 0.000008 \\
\hline 15 & 0.002926 & 0.000008 \\
\hline 16 & 0.002332 & 0.000008 \\
\hline 17 & 0.001856 & 0.000007 \\
\hline 18 & 0.001488 & 0.000006 \\
\hline 19 & 0.001197 & 0.000006 \\
\hline 20 & 0.000962 & 0.000007 \\
\hline 21 & 0.000775 & 0.000008 \\
\hline 22 & 0.000626 & 0.000007 \\
\hline 23 & 0.000504 & 0.000006 \\
\hline 24 & 0.000410 & 0.000006 \\
\hline 25 & 0.000335 & 0.000004 \\
\hline 26 & 0.000272 & 0.000004 \\
\hline 27 & 0.000222 & 0.000004 \\
\hline 28 & 0.000181 & 0.000005 \\
\hline 29 & 0.000146 & 0.000006 \\
\hline 30 & 0.000119 & 0.000005 \\
\hline 31 & 0.000098 & 0.000004 \\
\hline 32 & 0.000080 & 0.000003 \\
\hline
\end{tabular}

Table 7 


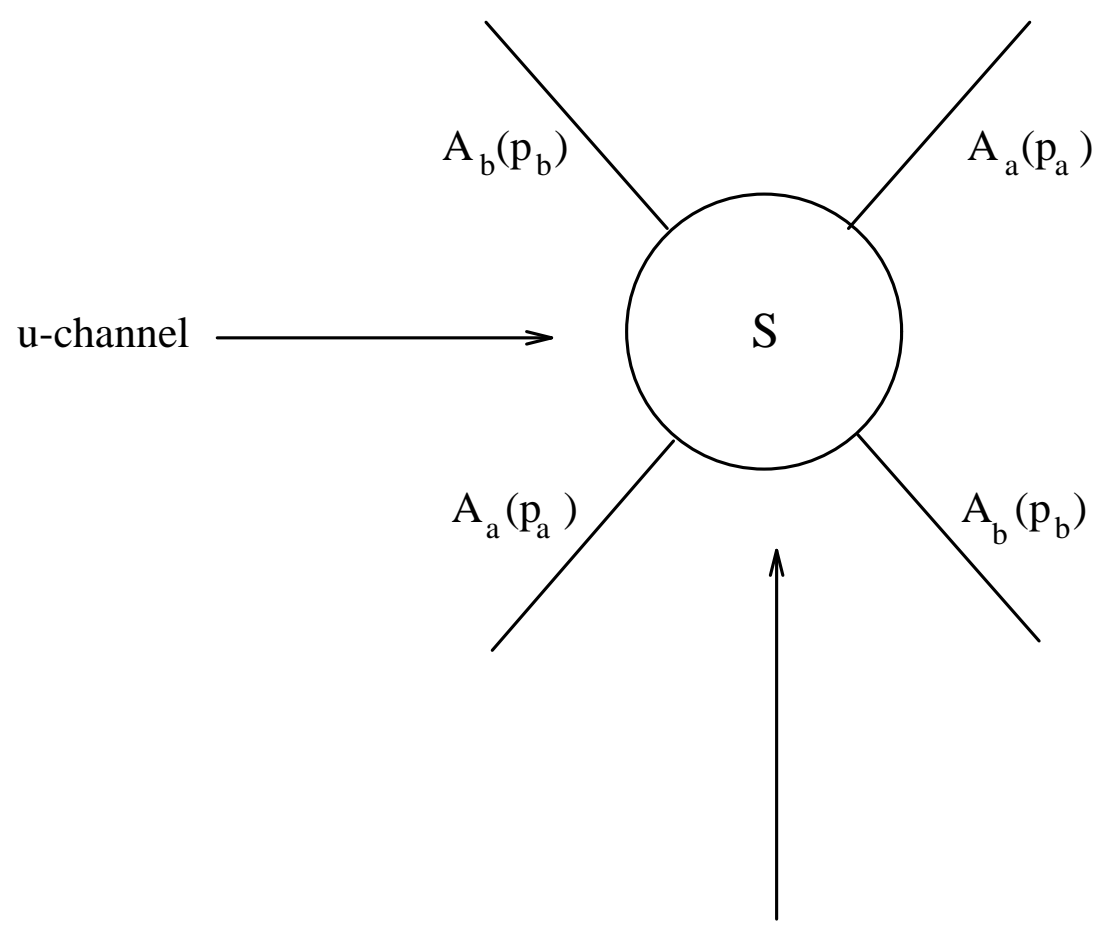

s-channel

Figure 1 


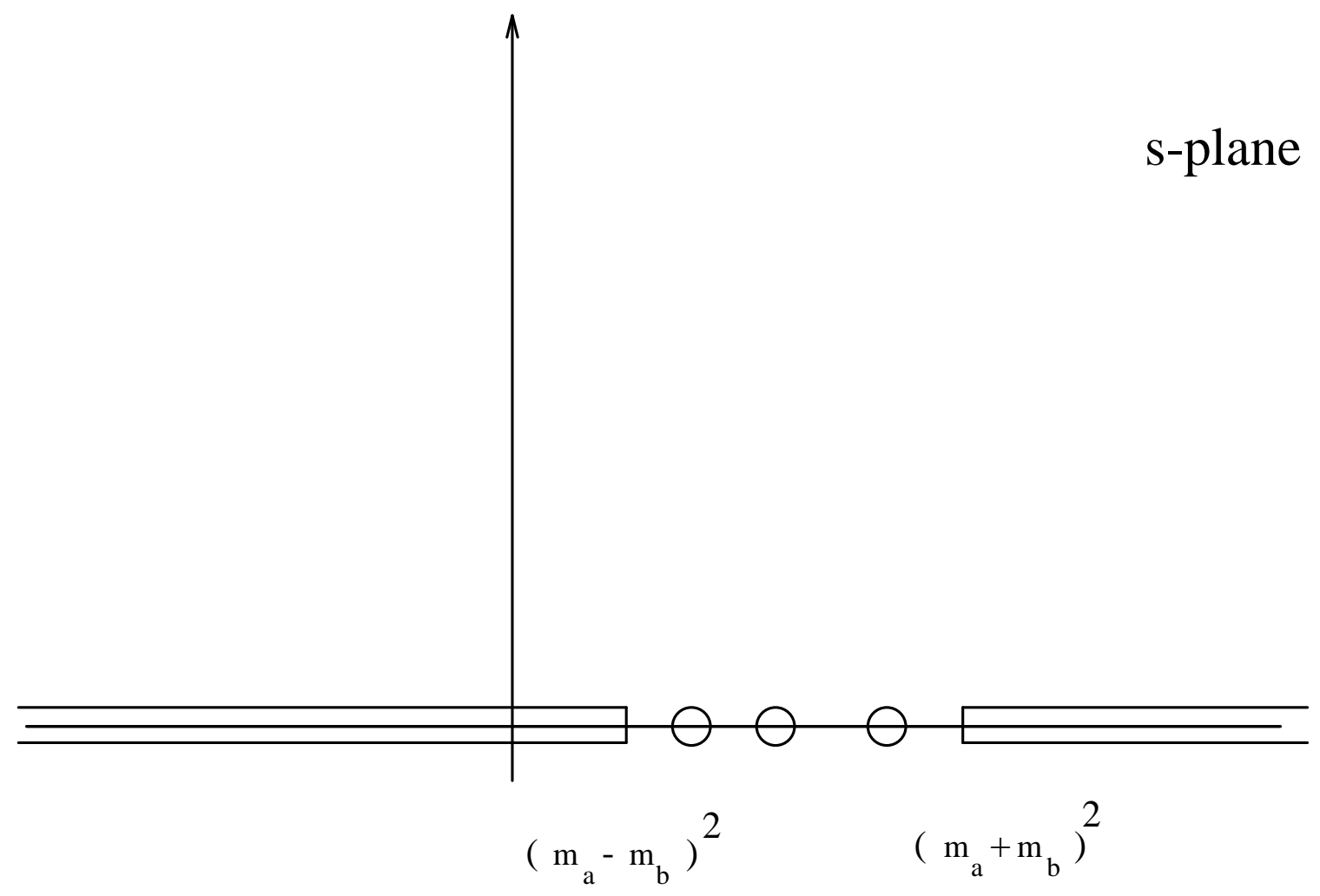

Figure 2 


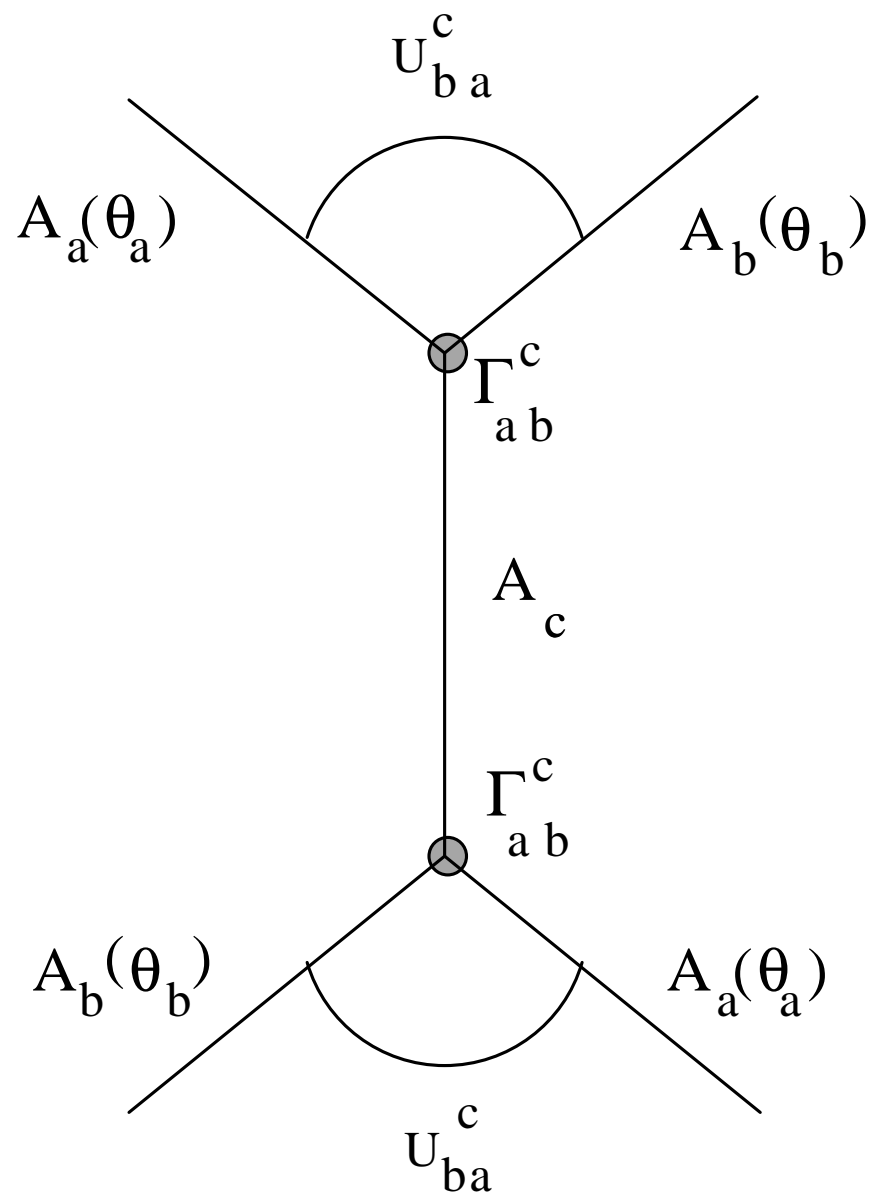

Figure 3 


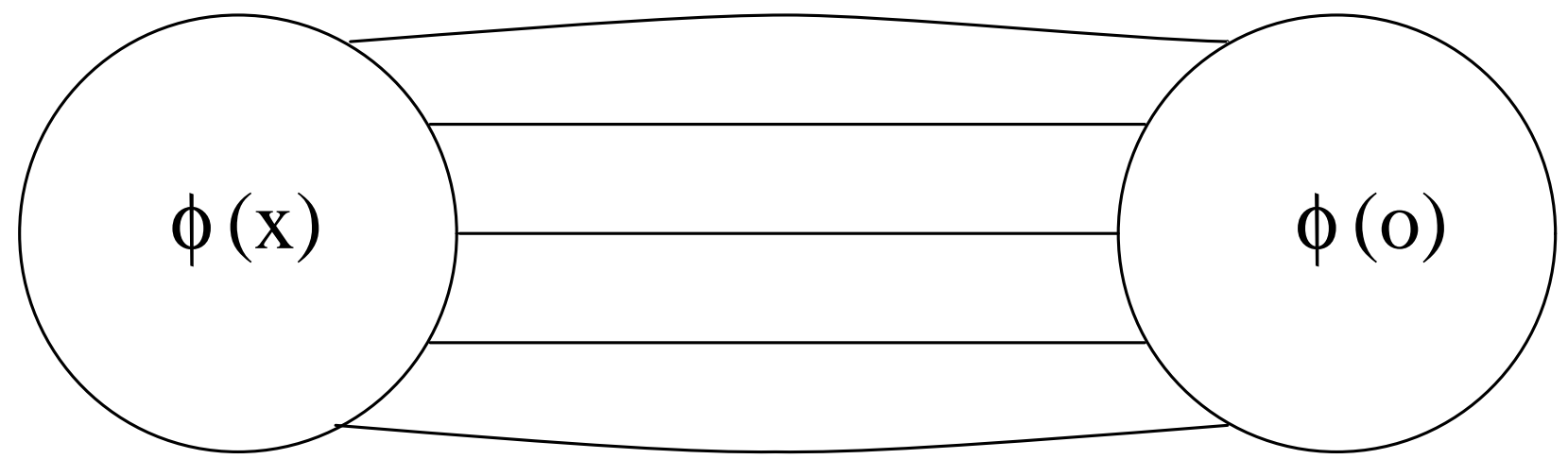

Figure 4 


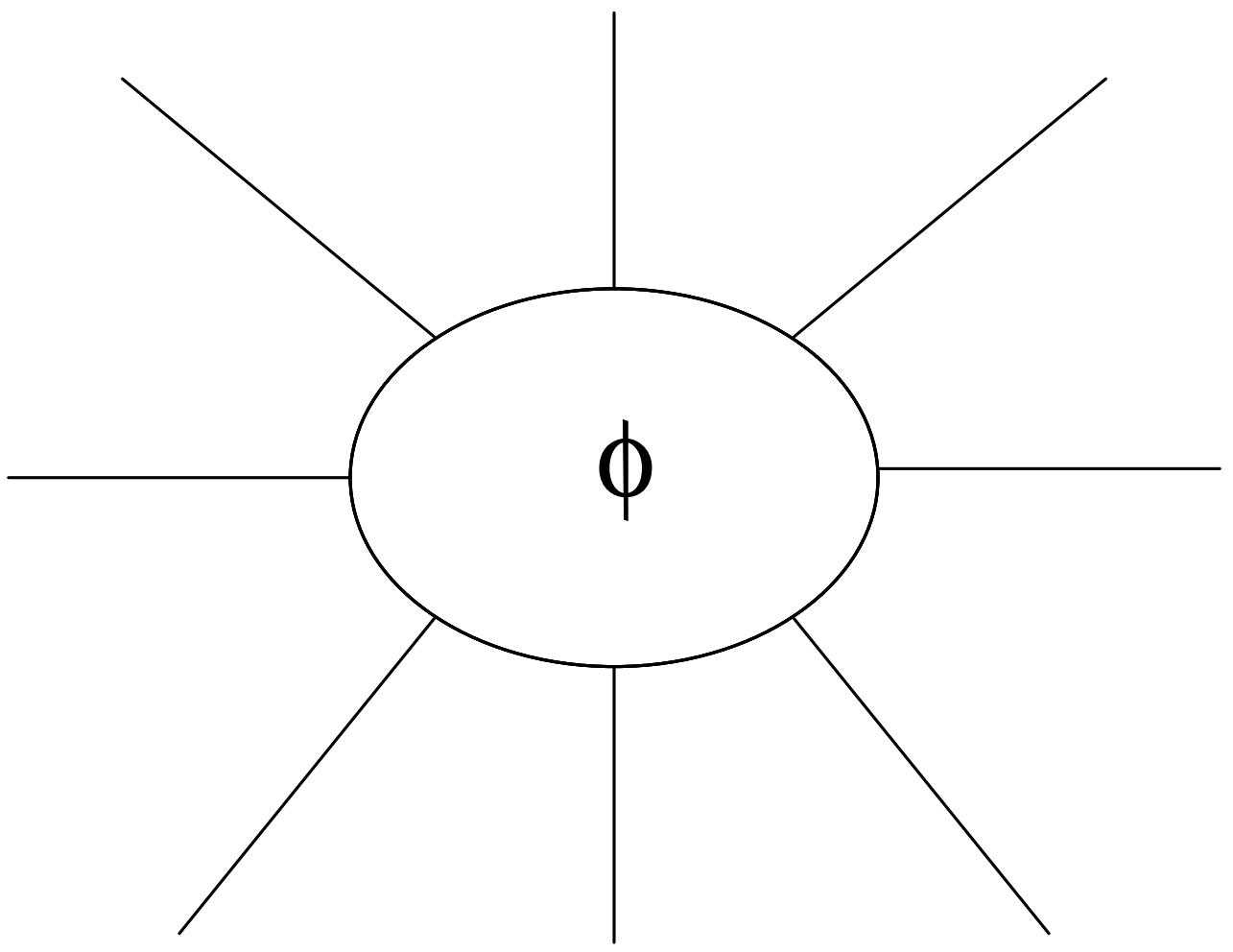

Figure 5 

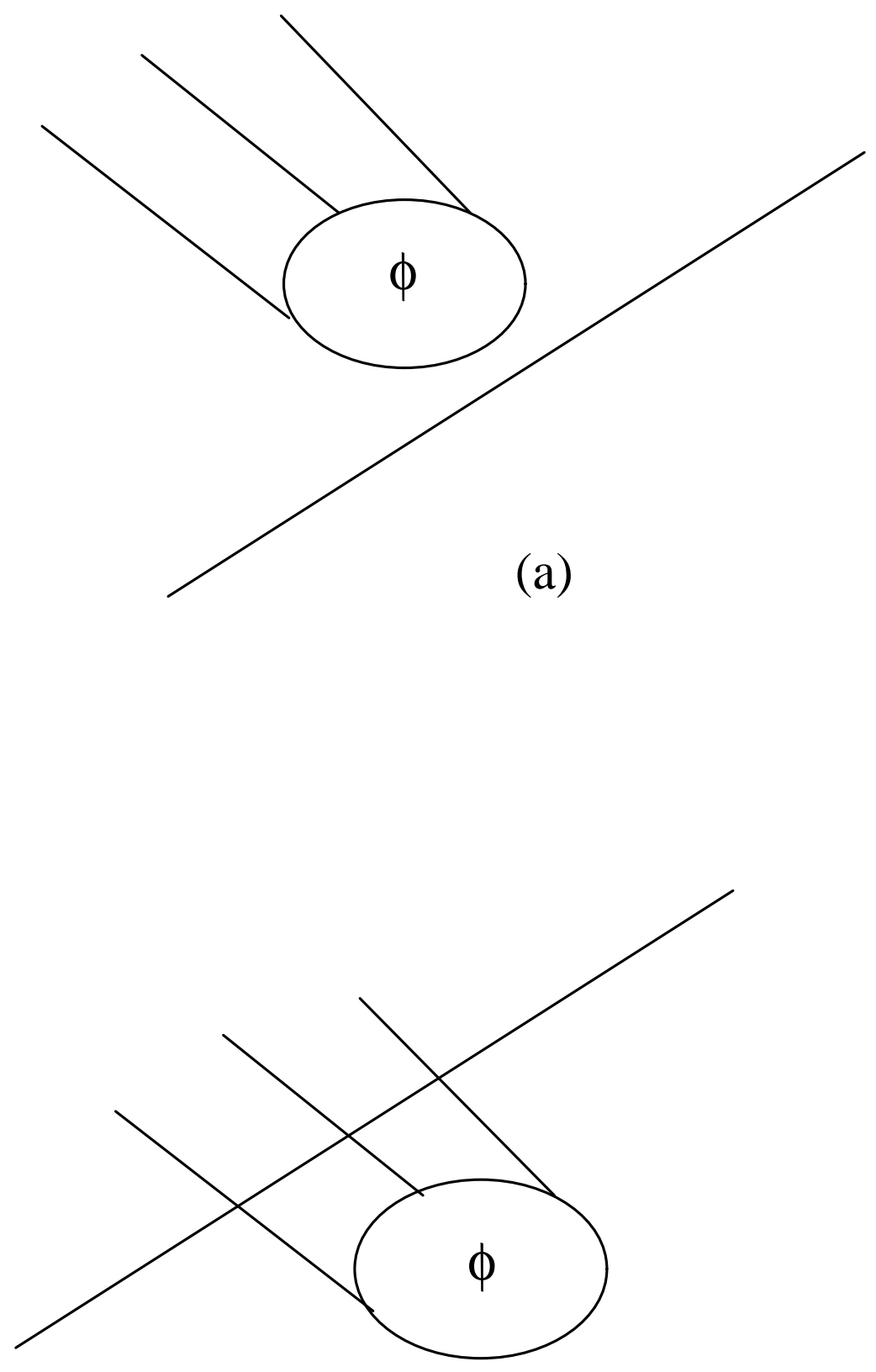

(b)

Figure 6 

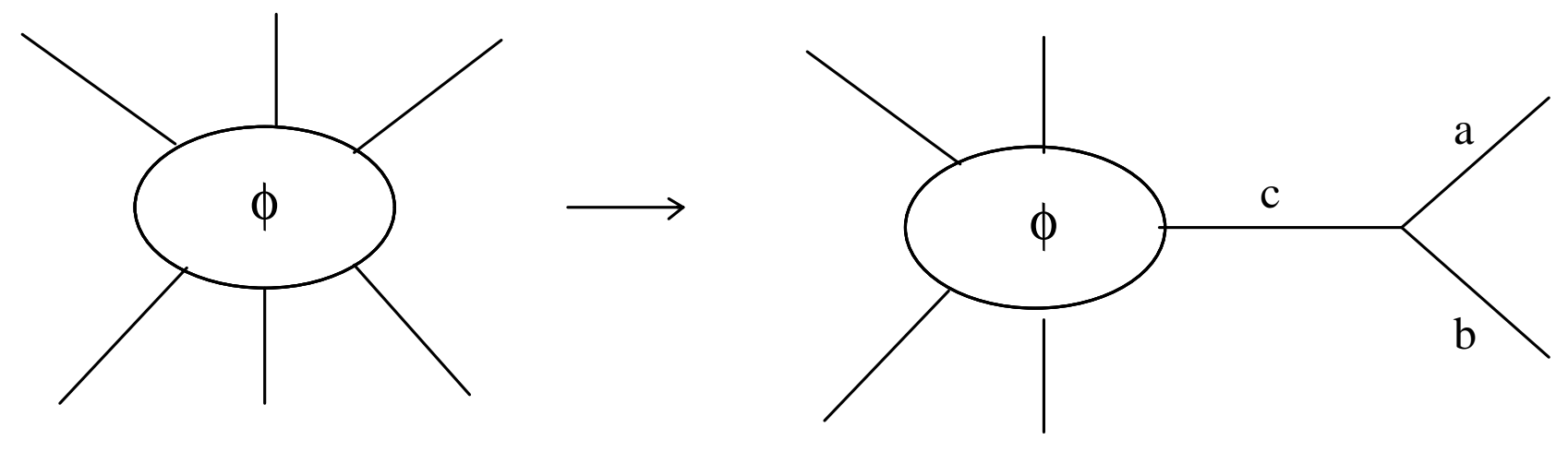

Figure 7 


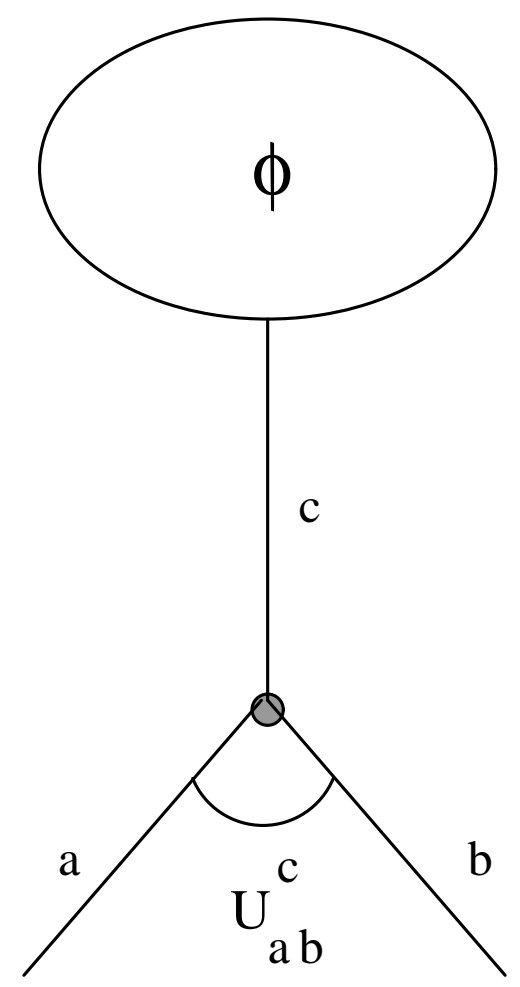

Figure 8 


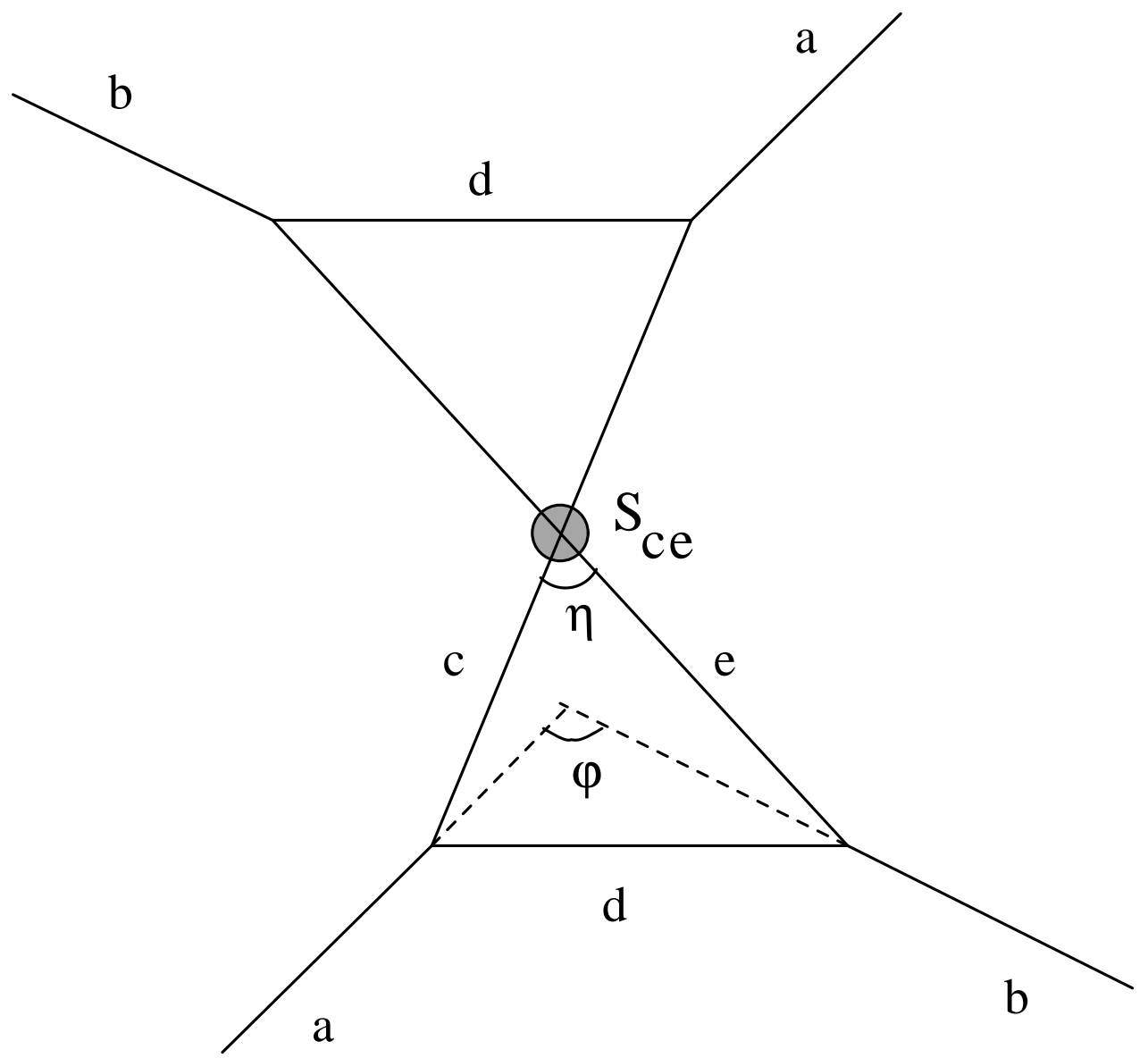

Figure 9.a 


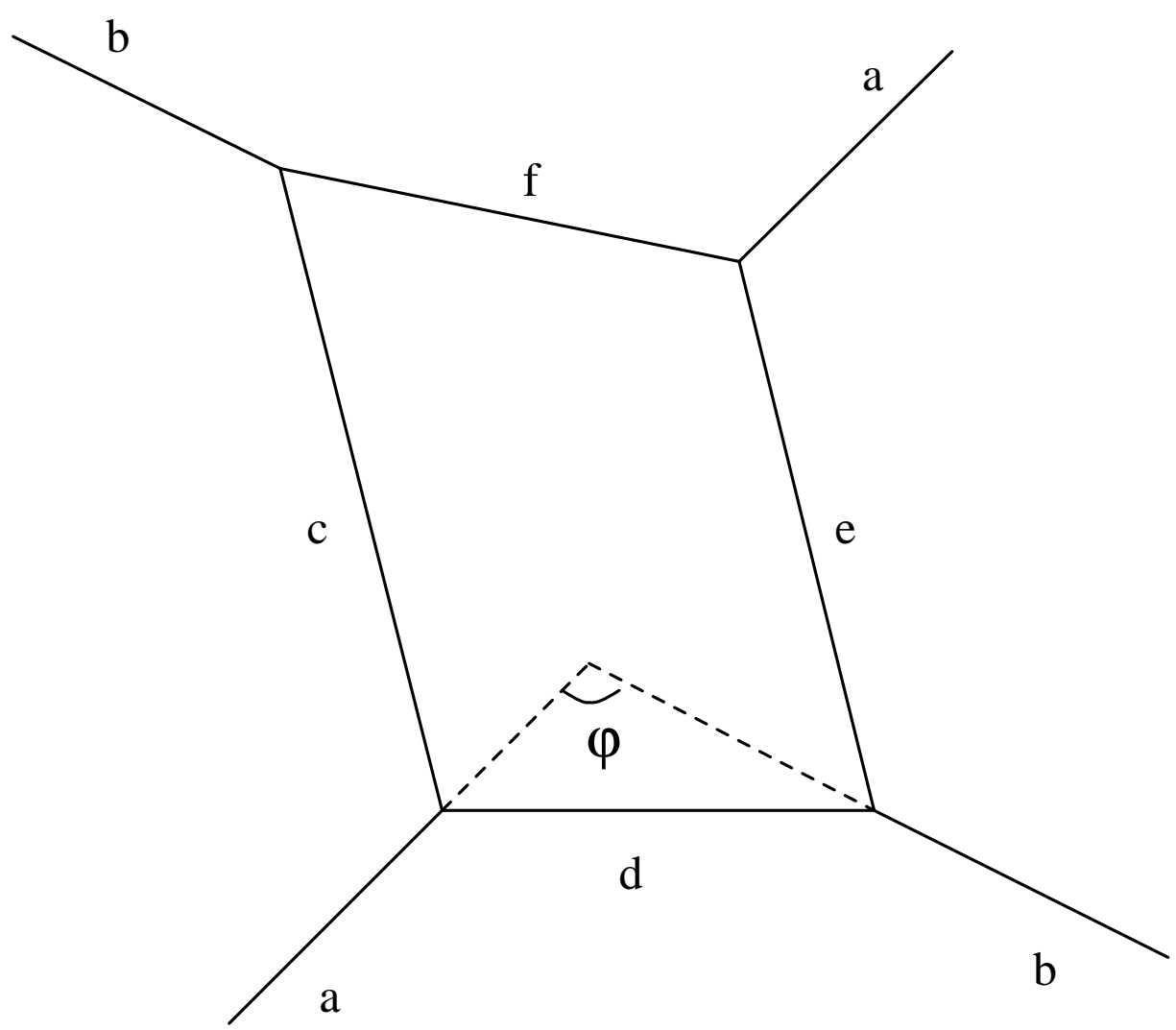

Figure 9.b 


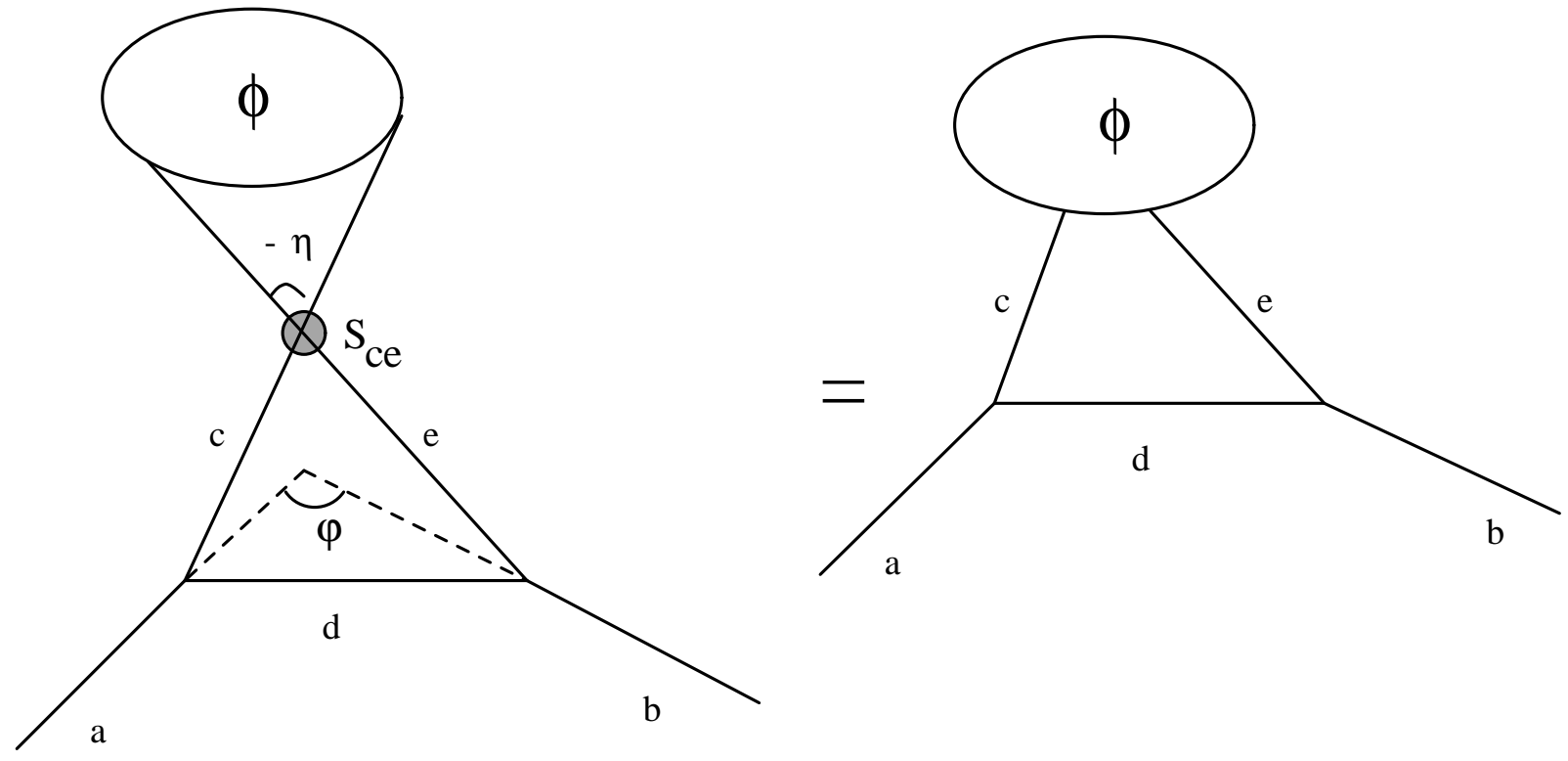

Figure 10 


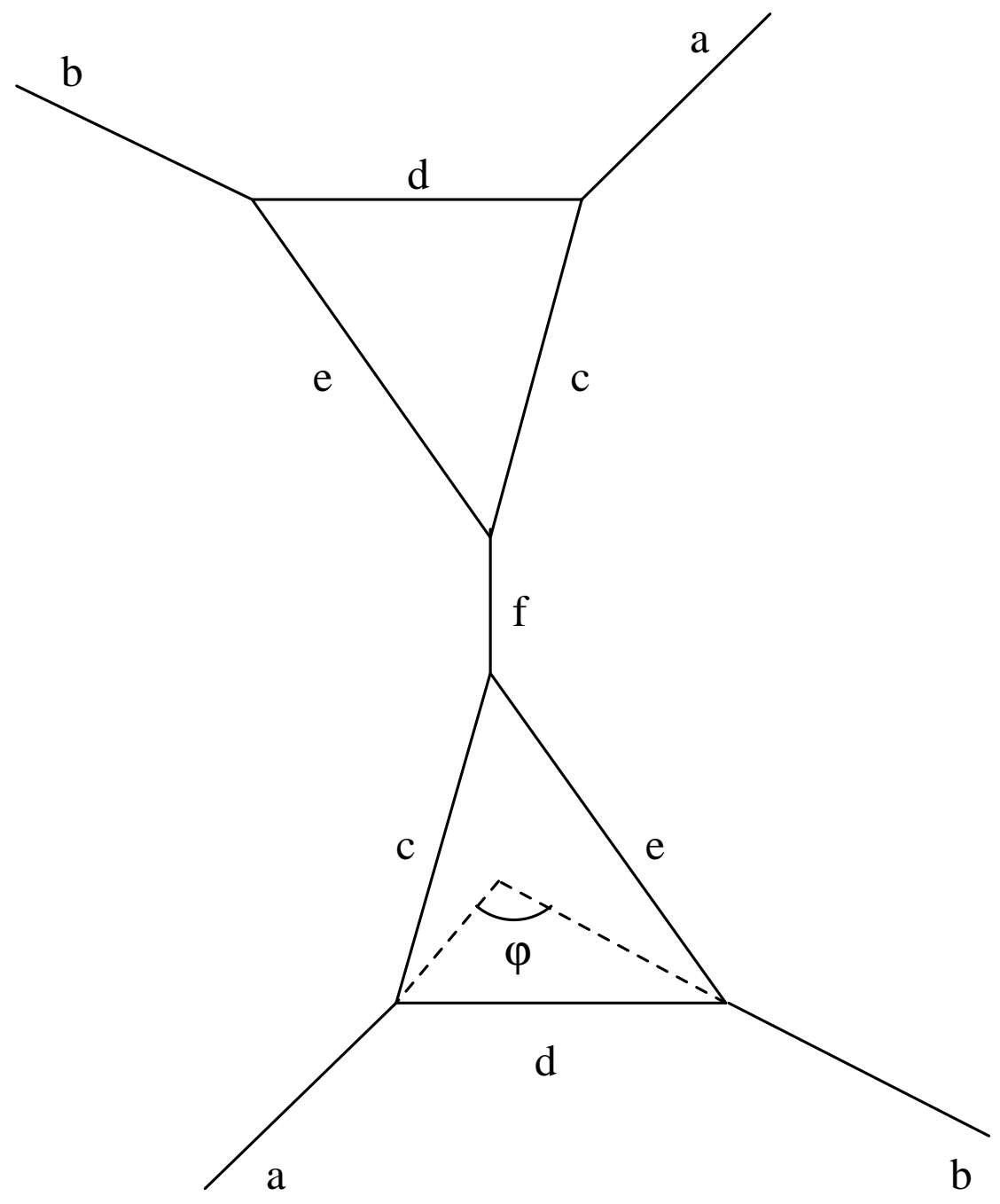

Figure 11 


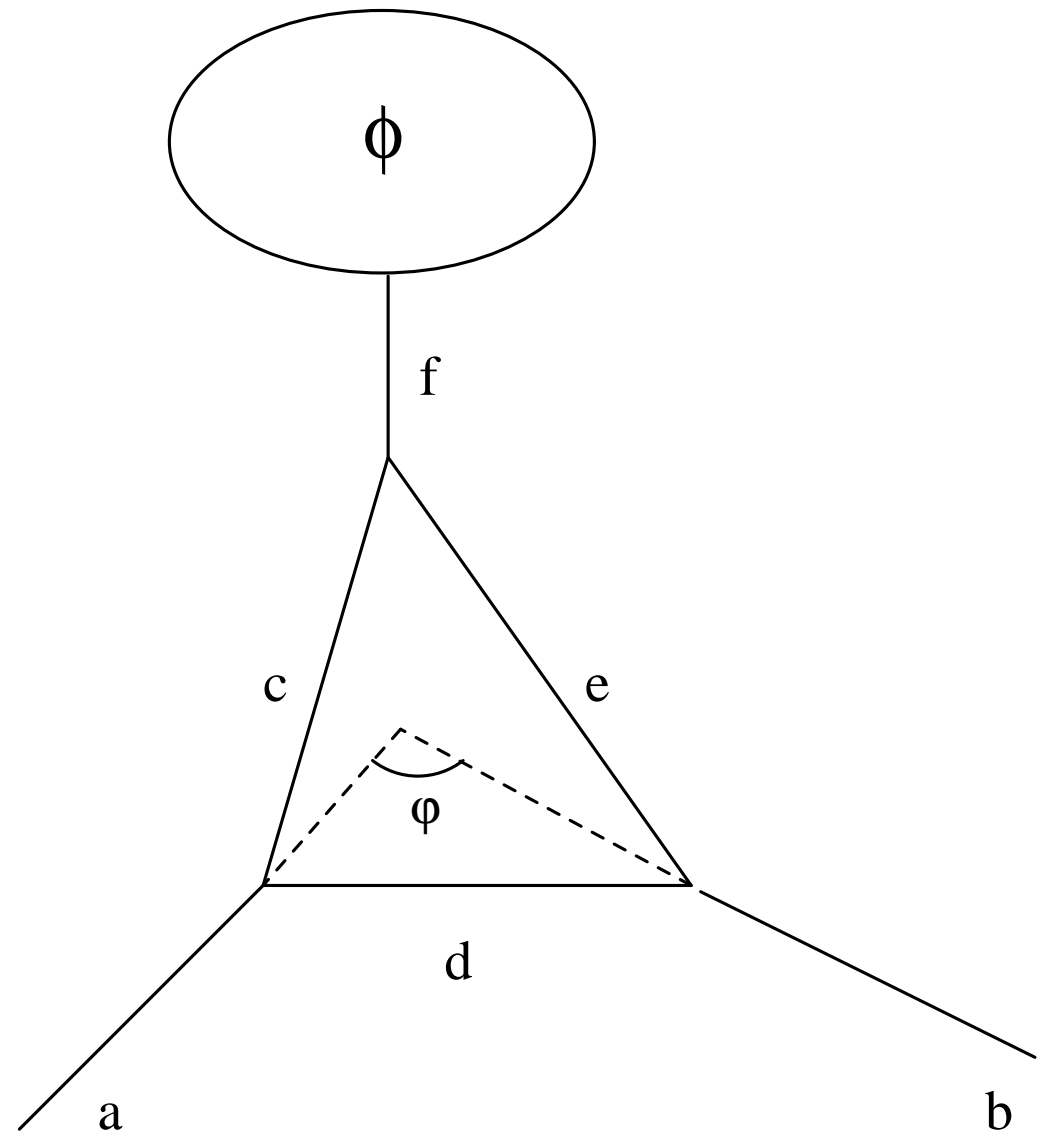

Figure 12 


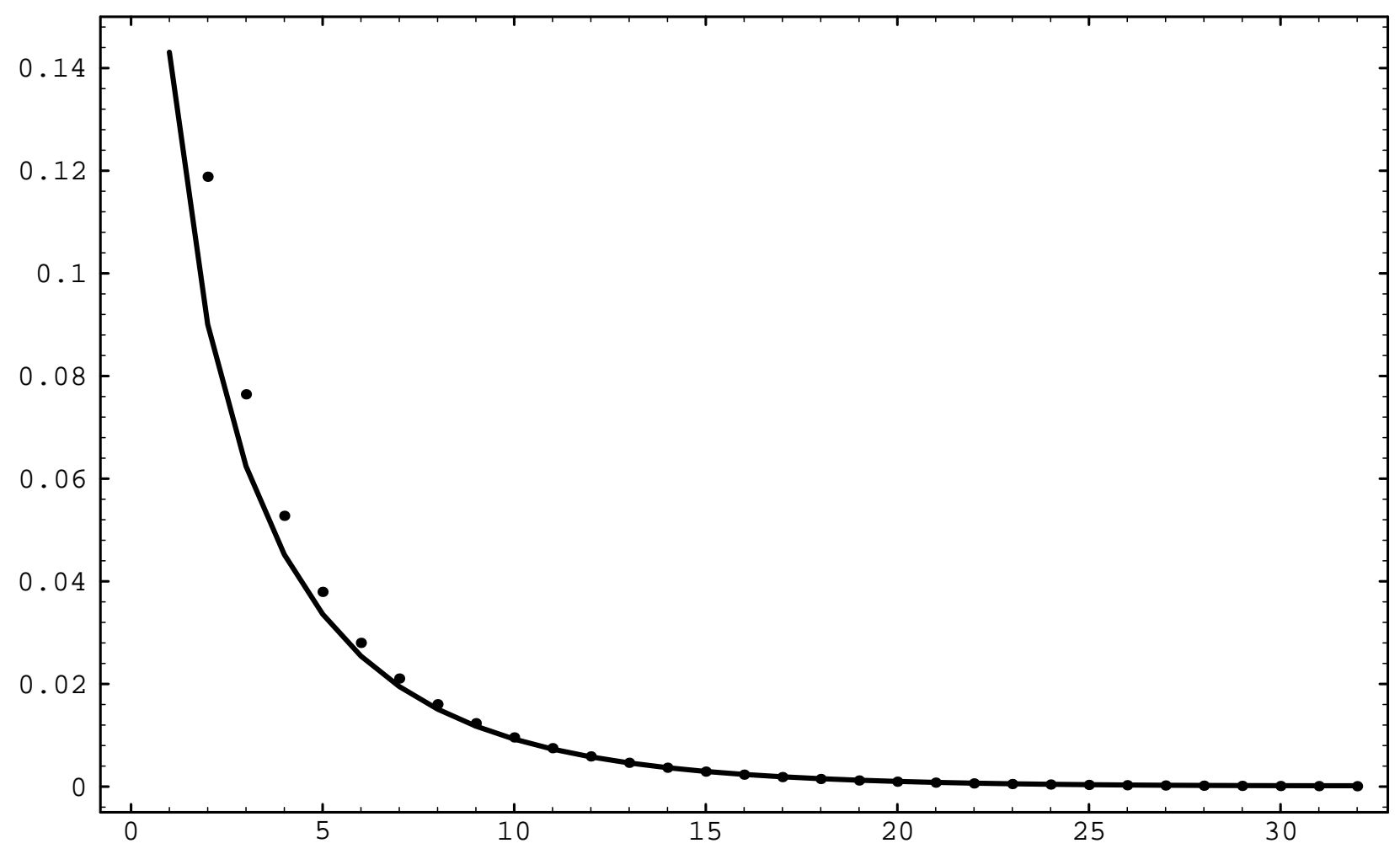

Figure 13 


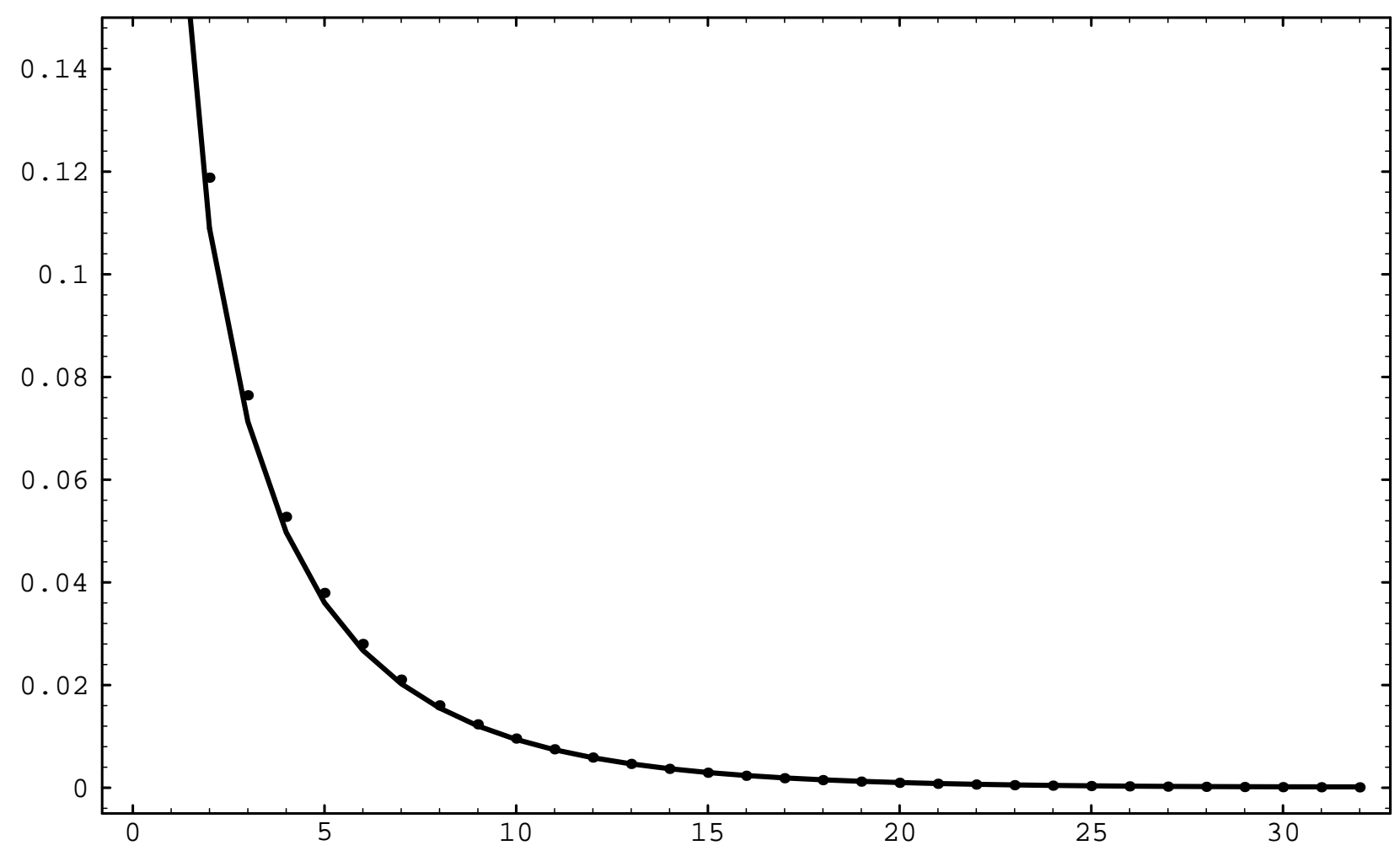

Figure 14 


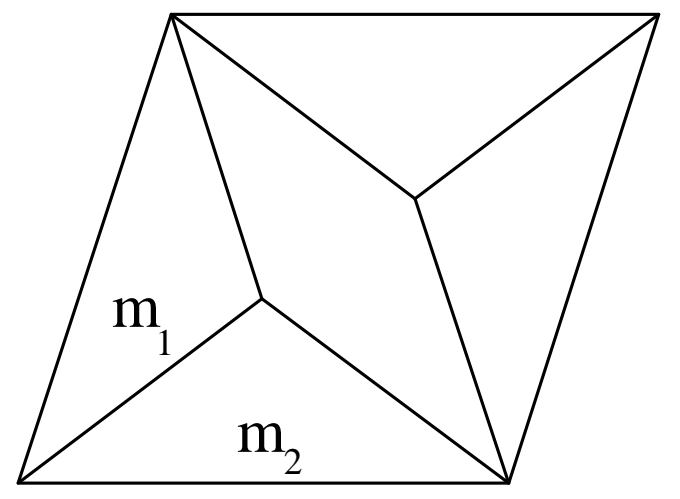

(a)

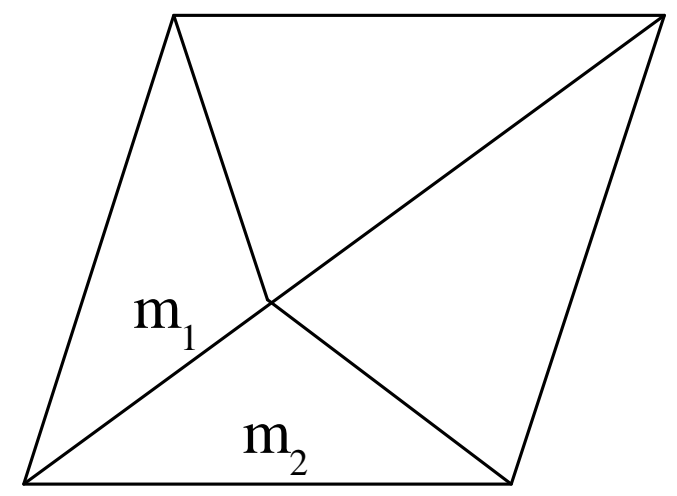

(b)

Figure 15 